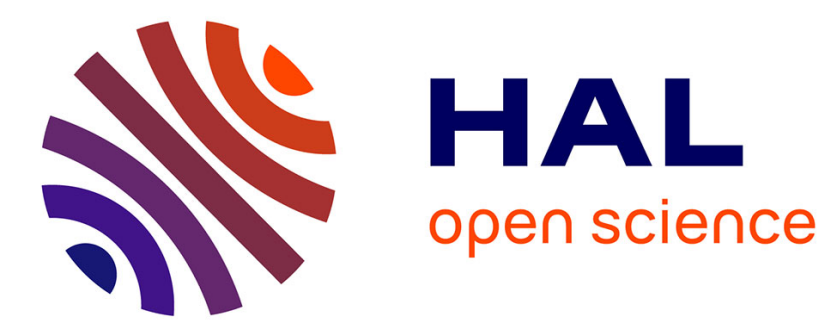

\title{
On broadband jet-ring interaction noise and aerofoil turbulence-interaction noise predictions
}

Michel Roger

\section{To cite this version:}

Michel Roger. On broadband jet-ring interaction noise and aerofoil turbulence-interaction noise predictions. Journal of Fluid Mechanics, 2010, 653, pp.337-364. 10.1017/S0022112010000285 . hal00566058

\section{HAL Id: hal-00566058 \\ https://hal.science/hal-00566058}

Submitted on 7 Jun 2012

HAL is a multi-disciplinary open access archive for the deposit and dissemination of scientific research documents, whether they are published or not. The documents may come from teaching and research institutions in France or abroad, or from public or private research centers.
L'archive ouverte pluridisciplinaire HAL, est destinée au dépôt et à la diffusion de documents scientifiques de niveau recherche, publiés ou non, émanant des établissements d'enseignement et de recherche français ou étrangers, des laboratoires publics ou privés. 


\title{
On broadband jet-ring interaction noise and aerofoil turbulence-interaction noise predictions
}

\author{
MICHEL ROGER† \\ Laboratoire de Mécanique des Fluides et Acoustique, École Centrale de Lyon, Ecully 69134, France
}

(Received 6 September 2007; revised 14 January 2010; accepted 14 January 2010;

first published online 5 May 2010)

The aerodynamic noise of a thin rigid annulus (referred to as the ring here) placed in the mixing layer of a subsonic circular jet is investigated in the paper, both theoretically and experimentally. From the experimental point of view, the jet-ring configuration is understood as an axisymmetric alternative to more usual ones involving a rectangular aerofoil held between parallel side plates, dedicated to the study of the noise due to the impingement of upstream turbulence. The main advantages of the circular geometry are a minimum background noise, the absence of tip effects and more specifically the account for all radiation angles from the surface in the far-field acoustic signature. The circular set-up is well suited for the study of pure broadband interaction noise only if the flow remains free of self-sustained oscillations. This is ensured by keeping a sufficient interaction distance between the nozzle and the ring, and by shaping serrations on the nozzle lip. From the theoretical point of view, an analytical model is derived as a straightforward extension of existing formulations. The induced unsteady lift forces on the ring are first inferred from a linearized unsteady aerodynamic theory and the far field is calculated in a second step by a radiation integral. This relates the far-field acoustic pressure power spectral density (PSD) to the two-wavenumber spectrum of the radial turbulent velocity at the ring location, by means of an aeroacoustic transfer function. The latter is shown asymptotically identical to the one detailed in the Appendix for a rectangular aerofoil, in the limit of relatively high frequencies. The analytical acoustic predictions are found to agree well with the measurements over an extended frequency range, provided that the model is fed with turbulent velocity input data measured by a hot-wire probe. Indirectly, this agreement validates the transfer function for a rectangular aerofoil at oblique radiation angles, which is not achievable in a set-up involving side plates and a rectangular nozzle.

\section{Introduction}

Noise radiation due to the impingement of upstream turbulence on a thin aerofoil is a generic mechanism involved in most topics of industrial interest, such as fan broadband noise, wake-interaction noise in turbomachines, high-lift device noise and so on. In order to meet more and more stringent noise reduction requirements, manufacturers generally need fast-running prediction methods that could be used extensively within a design cycle. Analytical techniques inherited from the pioneer work achieved by various authors in the seventies are still well suited for that goal,

$\dagger$ Present address: Centre Acoustique, École Centrale de Lyon, 36 Av. Guy de Collongue, 69134 Ecully, France. Email address for correspondence: michel.roger@ec-lyon.fr 
(a)

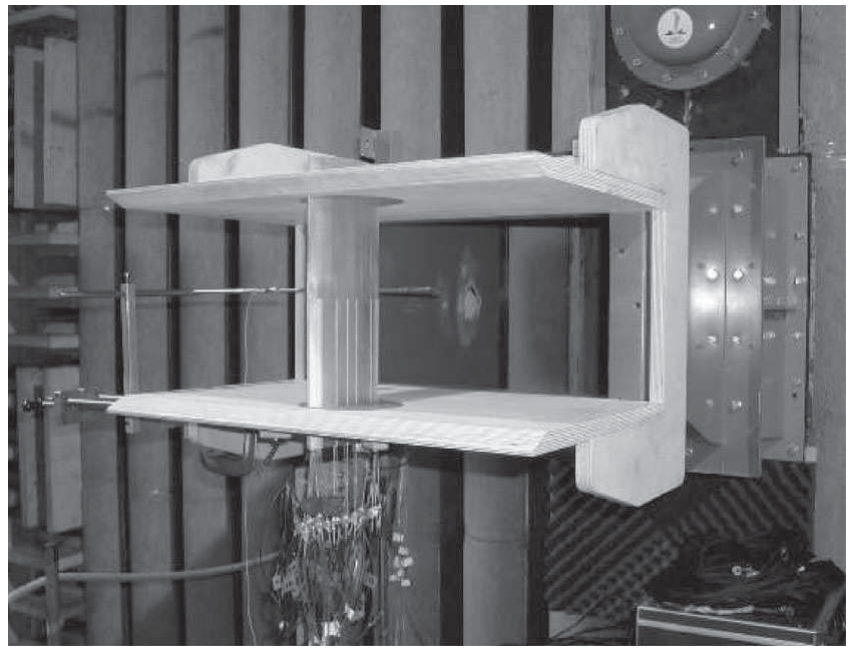

(b)

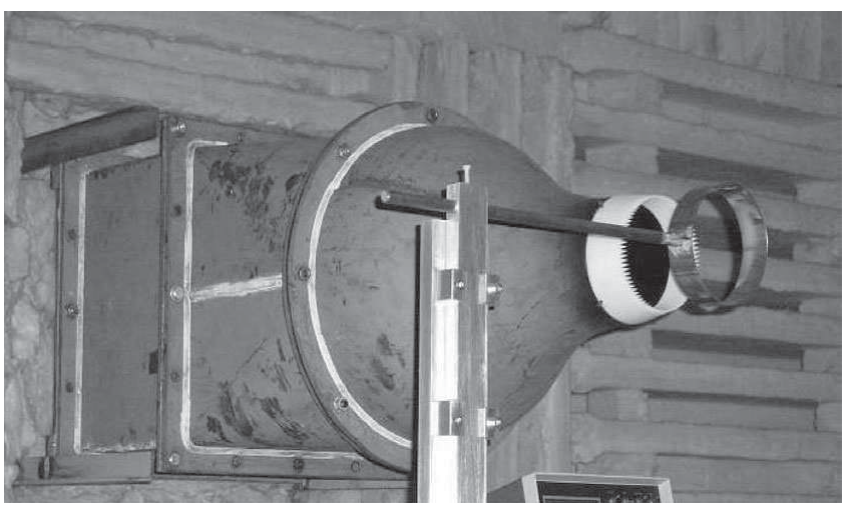

FIGURE 1. Pictures of the experimental set-ups in the open-jet anechoic wind tunnels of the École Centrale de Lyon. (a) Instrumented aerofoil mounted between horizontal side plates on a rectangular nozzle (Moreau \& Roger 2005). (b) Jet-ring arrangement, showing the sharp serrated nozzle lip in white and the horizontal strut holding the ring.

at least until the numerical methods become reliable enough at reasonable cost. In contrast they introduce drastic simplifications in the geometry and the flow field. For instance, the aerofoil is assimilated to a rigid flat plate with zero thickness and camber, and the mean flow is assumed uniform, at zero angle of attack. Therefore the closed-form solutions derived analytically must be carefully assessed against dedicated experimental studies for the sake of validation, before being used with confidence. Up to that point, the models do not aim at simulating all the very details of the aeroacoustic phenomena. On the one hand, they are just expected to reproduce the correct directivity patterns and spectral envelopes with typical errors not exceeding $3 \mathrm{~dB}$. On the other hand, they have to predict the correct changes with respect to variations of the main parameters of interest.

Previously published investigations refer to basic experiments performed in more or less anechoic open-jet wind tunnels of rectangular cross-section, with rectangular aerofoils held between side plates fixed to the nozzle lips (Paterson \& Amiet 1976; Moreau \& Roger 2005), as shown in figure 1(a). The aerofoil is embedded in the potential core of the undisturbed jet and additional incident turbulence is generated 
by an upstream grid located inside the nozzle. The angle of attack is easily varied by means of disks included in the plates and fixed to the aerofoil tips. Another possible set-up consists in placing directly the aerofoil in one of the lateral shearlayers of the wind-tunnel jet, as recently reported by Ross et al. (2008). The interest is to avoid the self-noise that would be generated by the turbulence grid. Several drawbacks are inherent to these rectangular-shape protocols. Firstly, noise can be unambiguously investigated only in the aerofoil mid-span plane and in the far field, because measurements off the mid-span plane would suffer from some masking or from sound scattering by the side-plate edges. As clearly pointed out by different authors (Amiet 1975; Paterson \& Amiet 1976), this geometrical constraint makes the space-Fourier components of the incident turbulence with phase surfaces parallel to the leading edge, called parallel turbulent gusts, contribute dominantly. The effect of the oblique gusts cannot be assessed because they radiate preferentially at oblique directions from the aerofoil surface. Consequently the experimental validation of theoretical response functions of rectangular aerofoils is at best incomplete. Secondly, the sound emitted from the aerofoil in the mid-span plane is scattered by the nozzle lips perpendicular to the side plates, and the measured directivity pattern slightly differs from the natural directivity corresponding to the direct aerofoil noise only. This is prejudicial to a clear comparison with the predictions and justifies the development of ad hoc corrections (Moreau, Schram \& Roger 2007). A third drawback is that the flow developing on the side plates generates a significant background noise, including trailing-edge noise at the plate ends, and a possible emission by the horseshoe vortices formed at the junctions between the aerofoil and the plates. For these reasons, boundary-layer suction devices, lined end-plates and trailing-edge serrations are possibly designed in some experimental set-ups in order to control the spurious noise sources, or arrays of microphones are used to focus on the investigated aerofoil noise sources (Brooks \& Humphreys 2003; Oerlemans \& Migliore 2004; Ross et al. 2008).

Other specific features of the usual rectangular set-ups are that the aspect ratio of the aerofoil $L / c, L$ being the span and $c$ the chord length, can be varied using aerofoils of various chord lengths, and that the turbulence parameters are defined by the grid design. Reliable results have been obtained this way in the past and more recently (Amiet 1975; Paterson \& Amiet 1976; Moreau \& Roger 2005), giving confidence to their use as a validation basis for analytical models. However, the validation off the mid-span plane is still missing. This is why some interest was found here to develop an alternative approach based on an equivalent experiment in circular geometry, taking all benefits of the axisymmetry of a circular jet, according to figure $1(b)$. In this experiment, the aerofoil is replaced by a thin annulus, typically a short slice of a hollow cylinder, referred to as the ring later on in the paper. The ring is placed in the mixing layer of the nozzle jet. The main advantages offered by this arrangement are listed below.

The first point is that all spurious noise sources associated with the side plates in the rectangular design are suppressed, since the ring is equivalent to a rectangular aerofoil that would have been curved so that its ends coincide. As a result, the frequency range over which the experimental results carry clear information about true turbulence-interaction noise is eventually extended.

The second point is about oblique radiation. Noise is symmetrically radiated around the axis, because both the geometry and the statistical properties of the turbulence in the jet mixing region are axisymmetric. However, the noise measured at a given location includes contributions from all possible angles of radiation from the surface 
with respect to the spanwise/azimuthal direction, due to the curvature of the ring. This provides a way of indirectly and globally assessing the effect of oblique radiation, a task not achievable with a rectangular-aerofoil set-up.

The third point is that the amount of scattering surfaces close to the real source to investigate is reduced to its minimum, so that the ring set-up is nearly free of spurious scattering effects.

It is worth noting that the statistical parameters of the turbulence interacting with the ring can be varied by changing the axial distance between the nozzle lip and the ring leading edge, or by using rings of different sizes. Here the major results are obtained with a reference ring which has the same diameter as the nozzle. Two other rings with diameters smaller and larger than the nozzle diameter by $20 \%$ are used occasionally for the sake of completing the study. In contrast the effect of the angle of attack cannot be easily investigated with the circular set-up. This limitation is not believed a serious drawback, because the angle of attack has been found to have a weak effect on the turbulence-interaction noise from a thin aerofoil in studies reported elsewhere (Paterson \& Amiet 1976; Moreau \& Roger 2005), at least within a range of moderate Reynolds numbers and of subsonic Mach numbers. A possible drawback of the circular set-up and of the rectangular set-up of Ross et al. (2008) is that the incident turbulence is not homogeneous and isotropic. This point will be discussed later on.

A major contribution of the paper is that the question of whether or not the conclusions of a validation study in circular geometry also hold for a rectangular aerofoil is positively answered, provided that some approximations are accepted. The main simplification underlying the analysis of $\S 3$ is that the streamwise aerodynamic transfer function between the velocity statistics in the oncoming flow and the induced lift fluctuations (function $\ell$ in the equations) is the same in both cylindrical and Cartesian coordinates. Intuitively, this is only true in the limit of a large curvature radius of the ring with respect to relevant scales in the linearized unsteady aerodynamics analysis, for instance, the azimuthal aerodynamic wavelength of the incident disturbances or the ring chord length. In that sense the present analysis is dedicated to sufficiently high frequencies. Practically the effective range of validity of the assumption will be determined by the final comparison between the model predictions and the experimental results. In fact the agreement will be found surprisingly good as shown in $\S 4$. It is to be noted that the use of a ring aerofoil in a jet to infer the noise from turbulence impinging on rigid obstacles has already been reported, for instance by Olsen (1976), only in the case of a compact chord length. The present study considerably extends the results by fully accounting for the effects of non-compactness, and by providing an accurate analytical model. Anyway, apart from its didactical interest and from validation purposes, the jet-ring tandem is also representative of configurations encountered in industrial applications, such as slotted nozzles, or the edge of the by-pass splitter cylinder in a turbofan engine swept by the turbulent wakes from the fan blades, or the blade-tip annulus of an automotive cooling fan ingesting turbulence. Finally, from the point of view of computational aeroacoustics, the jet-ring arrangement is a possible test case for the future validation of existing codes.

As a preliminary insight into well-known aeroacoustic mechanisms declined in circular geometry, the present study of jet-ring interaction noise is based on global simple experimental techniques such as far-field measurements using a single microphone and one-dimensional hot-wire anemometry. Furthermore the ring is not instrumented because of its small thickness $(1 \mathrm{~mm})$. More sophisticated techniques 
involving flush-mounted sensors, arrays of microphones for near-field modal analysis and cross-wire probes could be used in a further research. The set-up and far-field measurements are presented in $\S 2$ in order to emphasize on the main features of the sound field without going into the details. The self-sustained oscillations observed with the reference circular nozzle are mentioned but not detailed. They are suppressed by means of serrations on the nozzle to focus the study on pure broadband noise radiation. An analytical model dedicated to the broadband noise is derived in $\S 3$, based on linearized theories of unsteady aerofoil aerodynamics. In $\S 4$, the model equations are fed with the hot-wire measurements and the predictions are tested against the far-field data. A very good agreement is found, both in terms of directivity and frequency content.

\section{Experimental study}

\subsection{Experimental set-up}

The experimental set-up is shown in figure $1(b)$. The ring is held in the mixing layer of the jet by means of a small-diameter $(10 \mathrm{~mm})$ rod placed horizontally, normal to the jet axis. The rod is attached to a movable support that can be translated in the streamwise direction to vary the interaction distance $d$ between the nozzle lip and the ring leading edge. The nozzle diameter is $D=10 \mathrm{~cm}$ and three different rings with diameters $2 r_{0}=8,10$ and $12 \mathrm{~cm}$ and the same chord length $c=3 \mathrm{~cm}$ have been tested. The reference (middle-size) ring of diameter $2 r_{0}=D$ is always exactly facing the nozzle. The other two are used to investigate the response to turbulent eddies from the inner and outer layers of the mixing region as $d$ is increased. The exit flow speed $U_{0}$ ranges up to $60 \mathrm{~m} \mathrm{~s}^{-1}$, corresponding to a maximum Reynolds number based on the jet diameter $R_{e D}=U_{0} D / v=4 \times 10^{5}$, and a maximum chord-based Reynolds number $R_{e c}=U_{0} c / \nu=1.2 \times 10^{5}$. The results reported in this paper are obtained at $38 \mathrm{~m} \mathrm{~s}^{-1}$. Noise is measured in the horizontal plane containing the rod, at a distance $R_{m}=1.3 \mathrm{~m}$ from a reference point that coincides with the ring centre point when $d=D$. This ensures both acoustic and geometric far-field conditions in the sense that $2 \pi R_{m} \gg \lambda$ where $\lambda$ is the acoustic wavelength and $R_{m} \gg D$.

\subsection{Far-field results - effect of nozzle serrations}

The a priori knowledge of aeroacoustic mechanisms suggests that introducing a ring in a jet strongly increases the broadband noise radiation, because the impingement of the turbulence on the rigid surface of the ring generates dipole-like sources, which are much efficient than the quadruple-like sources of the jet-alone mixing noise, especially at subsonic Mach numbers (Curle 1955; Ffowcs Williams \& Hawkings 1969). Both sources are by-products of the same turbulence dynamics occurring in the flow region where the ring is introduced. The ring acts as a converter scattering a larger part of the kinetic energy of the turbulent field as sound. Thus only a strong enhancement of the contribution of that region to the jet mixing noise would be expected, over an extended frequency range. Actually, introducing the ring of diameter $2 r_{0}=D$ in the flow is found to result in the additional emission of high-intensity discrete-frequency tones for separations $d$ typically shorter than $1.5 D$ (shown in figure 2, dotted line).

The tones are an undesirable effect when resorting to the jet-ring set-up to investigate turbulence-interaction noise. Though not studied in the present paper, the corresponding flow regime has been characterized elsewhere by Roger \& Serafini (2005) for completeness, as a short contribution to the existing literature on selfsustained oscillations. The tones are attributed to an acoustic feedback mechanism 


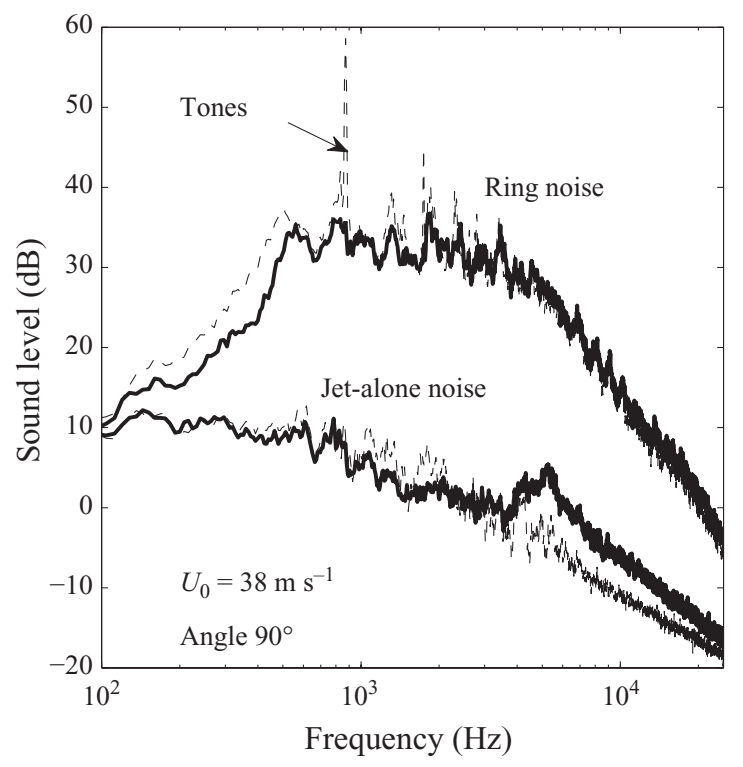

FIGURE 2. Typical far-field pressure PSD for jet-alone noise and ring noise, measured with the reference nozzle (dashed line) and with the serrated nozzle (dark continuous line). Short interacting distance $d / D=0.4$. $R_{m} / D=13$, observer at $90^{\circ}$ to the jet axis.

briefly outlined as follows (for a review of the literature on acoustic feedback phenomena, see Rockwell \& Naudascher 1978). The shear layer initiated at the nozzle lip oscillates according to instability modes, either axisymmetric (also called varicose modes) or non-symmetric (Schram 2003). These modes are amplified downstream and naturally give rise to shear layer roll-up, vortex pairing and three-dimensional breakdown, which cause the transition to turbulence. In a non-excited free jet at a sufficiently high Reynolds number, the transition extends over a very short length and the jet mixing layer turns fully turbulent quite close to the nozzle lip. The vortex pairing and instability mode dynamics do take place but they are randomly modulated. With an external properly tuned acoustic excitation, however, the mechanism is made more coherent (Schram 2003). In the present experiment with a regular nozzle and a short interaction distance, the forcing acoustic waves are provided by the ring itself. A growing instability mode induces acoustic sources when impinging on the ring. The corresponding acoustic wave propagating upstream is able to force the instability at its starting point close to the lip if the both of them are in phase relationship. The enhanced instability causes a stronger sound generation on the ring in return, and so on.

The feed-back loop must be broken in order to suppress the tones and observe more clearly the direct broadband radiation from the ring. This has been achieved here by empirically cutting the sharp-edged nozzle lip as a saw-tooth edge and increasing the interaction distance $d$. The serrations are about $8 \mathrm{~mm}$ deep and $3 \mathrm{~mm}$ wide, so that a hundred of them are distributed along the circular nozzle lip. Their effect on the noise spectra measured in the presence of the ring is highlighted in figure 2, continuous line. First, the serrations definitely suppress the tones and the ring-noise spectra turn fully broadband. Furthermore, below $600 \mathrm{~Hz}$, the broadband noise radiated by the ring is reduced by an amount of 3-6 dB with the serrated nozzle. Now the sound radiation involves both the amplitude and the azimuthal correlation length of the large-scale 
radial unsteady motion associated with the lower frequencies in the mixing layer. The saw-tooth cut is not expected to strongly reduce the amplitude at low frequencies. Therefore the sound reduction is attributed to a smaller correlation length. The jetalone noise obtained by just removing the ring spreads between 20 and $30 \mathrm{~dB}$ lower over a wide frequency range, though still well above the level measured when the wind tunnel is off, not shown here. The difference goes to zero only around the $100 \mathrm{~Hz}$ limit, where both jet noise and ring noise are lost in the background noise of the wind tunnel, and remains larger than $10 \mathrm{~dB}$ at the highest frequency investigated in the experiment, $25 \mathrm{kHz}$. The jet-alone noise is slightly modified with the serrated nozzle. An additional high-frequency hump around $5-10 \mathrm{kHz}$ is observed, as well as an increase of a couple of decibels above that range. This extra noise is attributed to aerodynamic sources located in the immediate vicinity of the serrations but not farther downstream. Indeed no evidence of a corresponding signature is found in the broadband spectrum measured with the ring installed. By the way, no noticeable change can be seen in the jet-alone noise spectrum below $600 \mathrm{~Hz}$ : the aforementioned reduced coherence of the initial large-scale motion has no apparent effect on the sound radiation of the reference jet.

Part of the jet-alone noise could be due to trailing-edge noise from the nozzle lip associated with the scattering of turbulent eddies convected in the boundary layers upstream of the nozzle. This contribution has not been quantified here with respect to the true mixing noise, the emphasis being on the noise radiated by the ring. In other words, the jet noise measured in the absence of the ring is considered the effective background noise in the present experiment dedicated to ring noise.

Figure 3 gathers an extended set of far-field measurements, the noise level being displayed as a function of frequency and angle of radiation, so that the two configurations with and without serrations can be compared globally. The directivity patterns of the self-sustained tones exhibit well-defined multiple lobes in the angular range covered in the experiment, between $20^{\circ}$ and $120^{\circ}$ from the jet axis. The broadband noise is more uniformly radiated, preferentially normal to the jet axis. The corresponding results will be compared to analytical predictions in $\S 4$.

\subsection{Mean-velocity profiles}

As a necessary step for a proper interpretation of the results, the mean flow profiles have been determined using Pitot measurements at different axial locations. Major results are plotted in figure 4 , referred to the radial location where the local speed is half the jet speed $U_{0}$ on the nozzle exit centreline. Figure 4(a) displays the nondimensional profiles measured closely downstream of the nozzle lip with the reference nozzle and the serrated nozzle. The serrations are found to make the free shear layer significantly thicker than what it would be with a regular nozzle. This is attributed to a radial flow developing around the serrations and responsible for some contamination of the undisturbed region, more fluid being dragged into the mixing layer. Since all the subsequent measurements are performed with the serrated nozzle, no comparison with the reference nozzle at farther distances is provided. The radial velocity profiles at downstream locations $d / D=2$ and $d / D=3$ are plotted in figure $4(b)$. They are almost independent of the flow speed, especially in the constant-shear middle part of the mixing layer where the ring of diameter $2 r_{0}=D$ is placed subsequently. The effective oncoming flow speed on the ring is around $60 \%$ of the speed $U_{0}$.

The measurements are well fitted by the theoretical hyperbolic tangent profiles

$$
\frac{U}{U_{0}}=\frac{1}{2}\left\{1-\tanh \left[\frac{D}{8 \delta^{*}}\left(2 y+\frac{2 y}{1+2 y}\right)\right]\right\}
$$



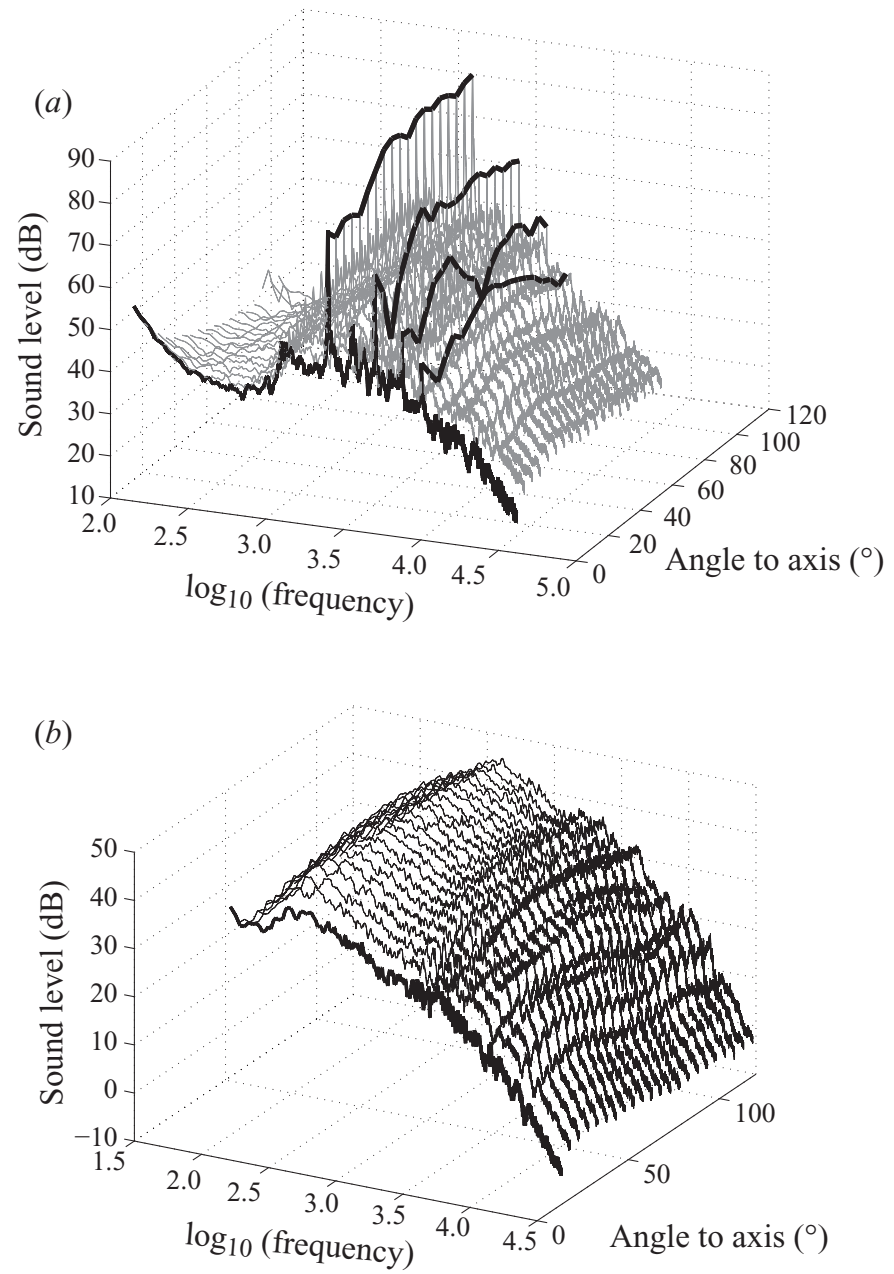

FIGURE 3. Typical angle-frequency plots of jet-ring interaction noise for directivity inspection. (a) Self-sustained oscillations with the reference nozzle $(d=4 \mathrm{~cm})$, directivity envelopes of the tones shown by thick lines. $(b)$ Broadband noise with the serrated nozzle $(d=20 \mathrm{~cm})$. $U_{0}=38 \mathrm{~m} \mathrm{~s}^{-1}, c=3 \mathrm{~cm}, r_{0}=5 \mathrm{~cm}, R_{m}=2 \mathrm{~m}$.
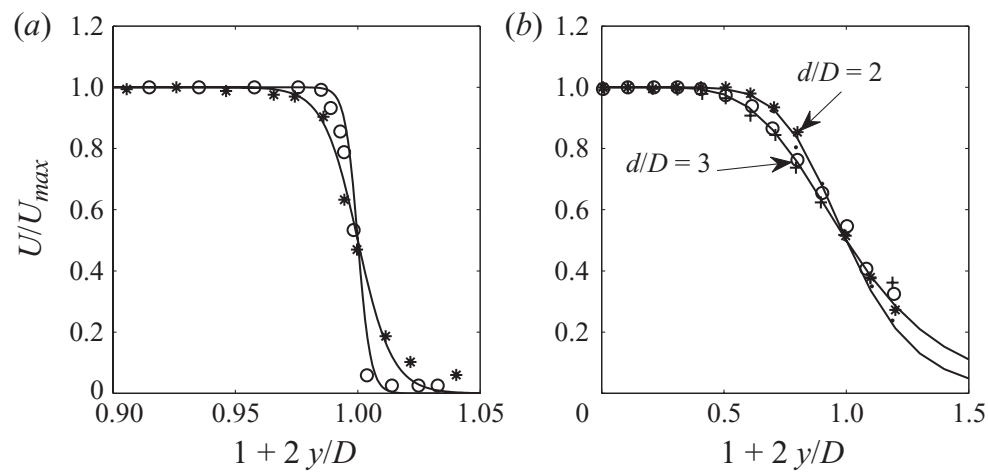

Figure 4. Mean-velocity profiles of the jet shear layers. (a) Close to the nozzle exit $d / D=0.03$ for the serrated nozzle (.) and for the reference regular nozzle (O) at $38 \mathrm{~m} \mathrm{~s}^{-1}$. (b) Farther downstream at $d / D=2$ and $d / D=3$ for the serrated nozzle and the velocities $38 \mathrm{~m} \mathrm{~s}^{-1}(\cdot,+)$ and $55 \mathrm{~m} \mathrm{~s}^{-1}(*, \bigcirc)$. 

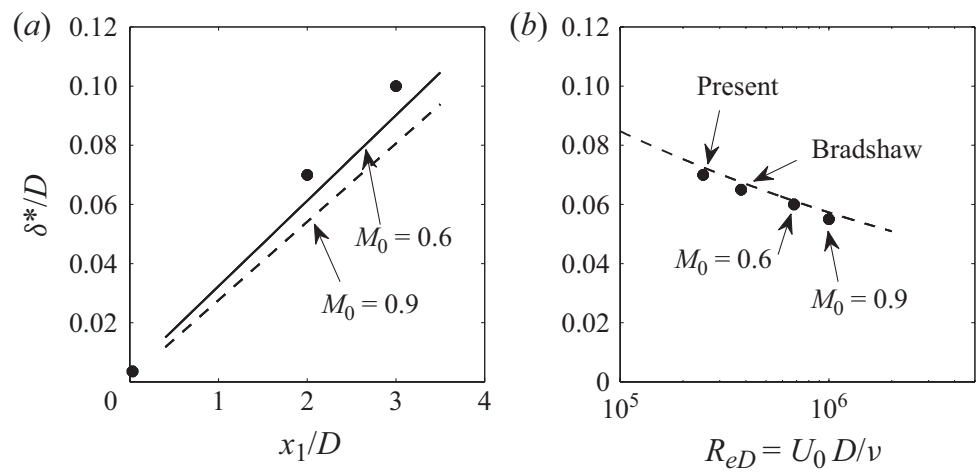

Figure 5. (a) Relative momentum thickness $\delta^{*} / D$ at $38 \mathrm{~m} \mathrm{~s}^{-1}$ in the present experiment (symbols), as a function of the axial location along the jet. Continuous and dashed lines stand for the measured trends from Fleury et al. (2008). (b) $\delta^{*} / D$ at $x_{1}=2 D$ as a function of the Reynolds number based on the diameter, including the data of Bradshaw et al. (1964).

in which $y$ is 0 for $U=U_{0} / 2$ and $y=-D / 2$ on the jet axis. $\delta^{*}$ denotes a momentum thickness of the shear layer tuned for a best fit at each axial location. The values of $\delta^{*}$ for the serrated nozzle are reported as symbols in figure 5(a) and compared to the trends measured by Fleury et al. (2008) on a jet from a regular nozzle of $5 \mathrm{~cm}$ diameter at the jet-exit Mach numbers $M_{0}$ of 0.6 and 0.9 , and the associated Reynolds numbers $R_{e D}=U_{0} D / v$ of $6.8 \times 10^{5}$ and $6.8 \times 10^{6}$. The momentum thickness is larger in the present case as expected from the lower flow speeds, corresponding to a Mach number of 0.11 and a Reynolds number of $2.5 \times 10^{5}$. The decrease of the relative thickness $\delta^{*} / D$ with the jet Reynolds number is emphasized in figure $5(b)$, also including an evaluation from the data reported by Bradshaw, Ferris \& Johnson (1964). The symbols follow a $R_{e D}^{-0.17}$ law, suggesting that the amount of shear is a slightly increasing function of the Reynolds number. The present results are in the continuation of previously reported studies, despite the different initial jet dynamics due to the serrations.

\subsection{Extended broadband noise measurements}

The measurements with the serrated nozzle, free of self-sustained oscillations, have been performed at different flow speeds, different interacting distances, with the three rings of diameters $0.8 D, D, 1.2 D$. To begin with, as shown in figure $6(a)$, for a fixed distance $d$, the noise level increases with the jet velocity $U_{0}$ and/or equivalently the local velocity at the ring radius $U$. The reduced acoustic PSD $S_{p p} / U_{0}^{5}$ are plotted in figure 6(b) as functions of the Strouhal number based on the jet diameter, $S_{t}=f D / U_{0}$. The results almost collapse, which indicates a scaling law for the mean square pressure in the far field with the sixth power of the local flow speed. The sixth-power law is expected from dimensional arguments, for compact dipoles at low speeds, provided that the oncoming velocity fluctuations are proportional to the mean flow speed. The last point is ensured by the self-similarity of the flow. The chordwise compactness condition expressed on the Helmholtz number ignoring the convection effect reads $k c \ll 1$, where $k=\omega / c_{0}$ is the acoustic wavenumber and $c_{0}$ the speed of sound. It holds reasonably for frequencies lower than, say, $1700 \mathrm{~Hz}$. The same condition in the radial direction reads $k D \ll 1$ and is fulfilled at frequencies lower than $500 \mathrm{~Hz}$. Thus it is not surprising that the best fit with the sixth-power law in figure $6(b)$ is achieved at the low Strouhal numbers $\left(S_{t}=f D / U<1\right)$ roughly corresponding to the rising slope 


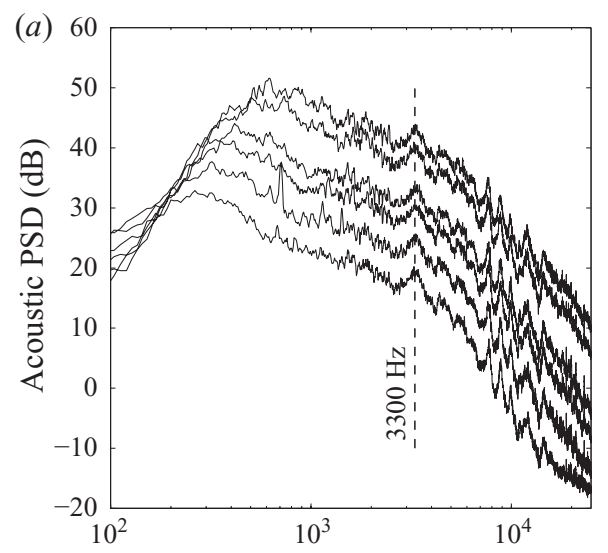

(b) $\quad$ Frequency $(\mathrm{Hz})$

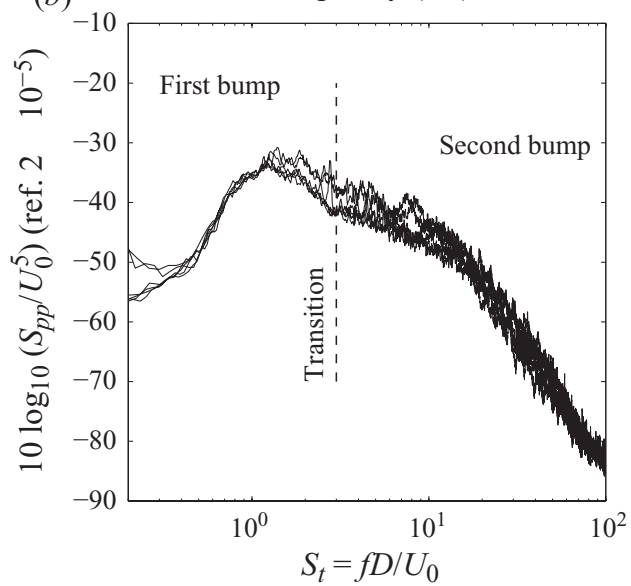

FIgURE 6. (a) Typical measured sound spectra at $90^{\circ}$ to the flow, for the reference ring of radius $r_{0}=D / 2$ and an interaction distance $d=2 D$, at various flow speeds $(21.4,25.6,31.1$, $34.8,39.5,44.4) \mathrm{m} \mathrm{s}^{-1}$ corresponding to increasing loudness. (b) Approximate data collapse according to the sixth-power law.

of the low-frequency bump. More scattering is observed on the high-frequency part of the spectra. Larger discrepancies are also seen in the middle range $1<S_{t}<10$. Some of them are due to the slightly different transition frequencies between the dominant bump and the secondary one at different flow speeds. Some other, such as the small peak around $3300 \mathrm{~Hz}$ visible on all plots of figure $6(a)$ and the series of spikes and small-amplitude oscillations at higher frequencies, might be attributed to residual resonant frequencies inherent to the installation. Typically, successive reflections of sound waves between ring surface elements diametrically opposed are likely to produce such patterns. The small oscillations will not be addressed in the analysis.

The noise spectra measured with the three different rings in the same jet crosssection at the exit flow velocity of $38 \mathrm{~m} \mathrm{~s}^{-1}$ are plotted in figure $7(a)$. The radiated noise is not very different with the rings of radii $r_{0}=4,5 \mathrm{~cm}$, except for an increased level at high frequencies for the smallest. In contrast, the spectrum measured with the larger ring $\left(r_{0}=6 \mathrm{~cm}\right)$ drops more rapidly in the high-frequency range. This is a joint effect of the radial variations of the local mean flow speed and of the local 

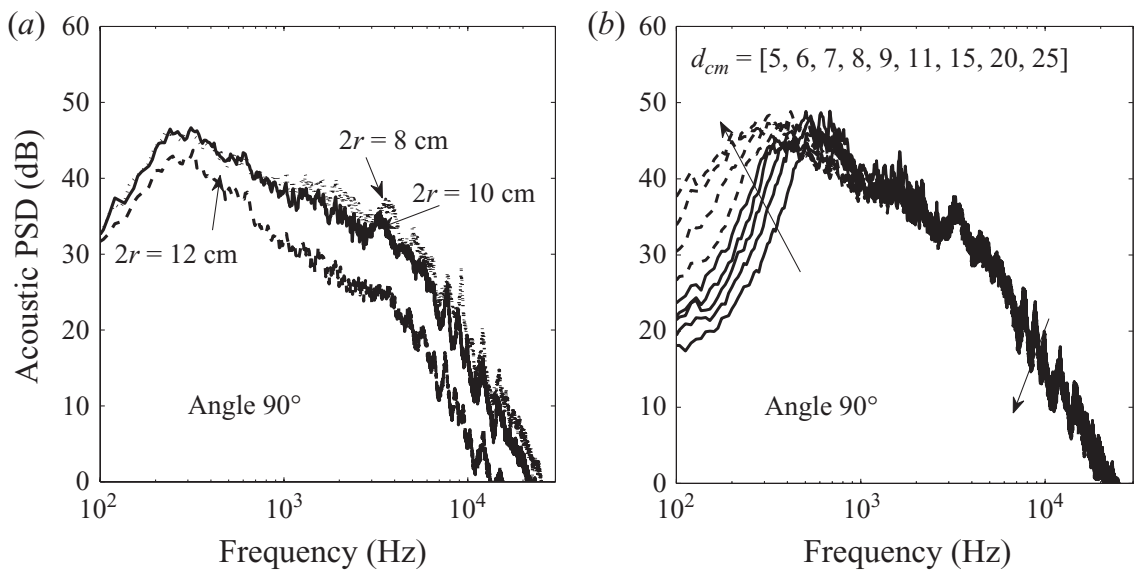

FIGURE 7. Effect of ring size and of interaction distance $d$ on broadband jet-ring interaction noise. $U_{0}=38 \mathrm{~m} \mathrm{~s}^{-1}$. (a) $d=2 D$, different ring sizes. (b) Ring $2 r_{0}=D$, different separations $d$ in $\mathrm{cm}$. The arrows indicate increasing distance.

rate of fluctuation. Points closer to (respectively farther from) the jet axis correspond to a higher (resp. lower) speed and to higher (resp. lower) frequencies for the same eddy size of the convected turbulence. The velocity profiles of figure 4 show that because of the jet expansion, the larger and smaller rings placed at the downstream distance $2 D$ approach the boundaries of the mixing layer. Therefore the locations of the leading and trailing edges of the rings correspond to very different flow conditions. The acoustic signatures of figure 7(a) cannot be interpreted unambiguously with the analysis of $\S 3$. Yet the smaller and larger rings will be used later on to assess the limitations of the prediction model.

Finally, noise spectra measured at different axial locations with the reference ring $\left(r_{0}=D / 2\right)$, for the same jet velocity, are shown in figure $7(b)$. As the ring is moved away from the nozzle lip, the noise level radiated in the low-frequency range increases significantly, whereas the middle-and-high frequency part of the spectrum decreases of only a couple of decibels. At the radial ring location facing the lip, and for the range of values of $d$ investigated, the local mean flow speed is nearly constant (see figure 4). Therefore the different acoustic radiation is mainly attributed to the formation of larger eddies; small-scale turbulence is rather distributed more uniformly.

\section{Broadband noise predictions}

\subsection{Analytical model}

In the model, the ring is assumed perfectly rigid and with zero thickness. Its chord length is $c$ and its diameter $D=2 r_{0}$. The origin is at the ring centre point. A source point on the ring surface is defined either by its Cartesian coordinates $\boldsymbol{y}=\left(y_{1}, y_{2}, y_{3}\right)$ or by the cylindrical coordinates $\left(y_{1}, \theta\right)$ with reference at the mid-chord perimeter, shown on the sketch of figure 8 . The observer is in the acoustical and geometrical far field, thus at large distances when compared to both the acoustic wavelengths and the ring radius. His location is defined by the coordinate vector $\boldsymbol{x}=\left(x_{1}, x_{2}, x_{3}\right)$. The contribution of a ring element to the far-field sound is first assimilated to the noise from a point dipole in a fluid moving uniformly with the velocity $U$, here along the $x_{1}$ direction, $U$ being the local mean velocity at the exact radius where the ring lies in the jet. This simplification will be discussed later on. The contribution of the point 


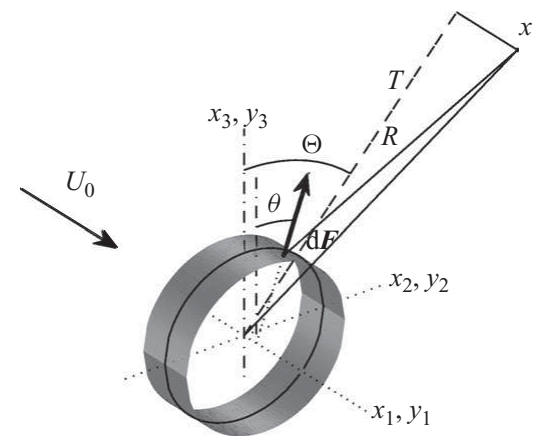

Figure 8. Set of coordinates for ring noise calculations. The oncoming flow is in the $x_{1}$ direction.

dipole to the acoustic pressure reads

$$
\mathrm{d} p^{\prime}(\boldsymbol{x}, t)=\frac{\boldsymbol{R}_{t}}{4 \pi c_{0} R_{s}^{2}} \cdot\left[\frac{\partial(\mathrm{d} \boldsymbol{F})}{\partial t^{\prime}}\right]_{t-R_{t} / c_{0}},
$$

where $\boldsymbol{R}_{t}$ is the modified distance vector $\left(\left(R_{1}-M R_{s}\right) / \beta^{2} ; R_{2} ; R_{3}\right)$, with $\boldsymbol{R}=\boldsymbol{x}-\boldsymbol{y}$, $R_{s}^{2}=\left(x_{1}-y_{1}\right)^{2}+\beta^{2}\left[\left(x_{2}-y_{2}\right)^{2}+\left(x_{3}-y_{3}\right)^{2}\right]$ and $\beta^{2}=1-M^{2}, M=U / c_{0}$ being the Mach number based on a constant sound speed $c_{0}$. The corresponding distance reads $R_{t}=\left(R_{s}-M\left(x_{1}-y_{1}\right)\right) / \beta^{2}$. The ring element of area $\mathrm{d} S$ is the support of a fluctuating force $\mathrm{d} \boldsymbol{F}$ corresponding to the local unsteady lift per unit area $\ell$ induced by the oncoming disturbed flow: $\mathrm{d} \boldsymbol{F}=\ell\left(y_{1}, \theta, t\right) \boldsymbol{n} \mathrm{d} S, \boldsymbol{n}$ standing for the normal unit vector pointing outwards. The far-field assumption yields simplified expressions for the corrected distance $R_{s}$ :

$$
\begin{aligned}
& R_{s} \simeq S_{0}\left(1-\frac{x_{1} y_{1}+\beta^{2} r_{0}\left[x_{2} \sin \theta+x_{3} \sin \theta\right]}{S_{0}^{2}}\right), \\
& S_{0}^{2}=x_{1}^{2}+\beta^{2}\left(x_{2}^{2}+x_{3}^{2}\right), \quad \frac{1}{R_{s}^{2}} \simeq \frac{1}{S_{0}^{2}} .
\end{aligned}
$$

With the aforementioned conventions, the elementary acoustic field is written as

$$
\mathrm{d} p^{\prime}(\boldsymbol{x}, t)=\frac{R_{2} \sin \theta+R_{3} \cos \theta}{4 \pi c_{0} S_{0}^{2}}\left[\frac{\partial(\mathrm{d} F)}{\partial t^{\prime}}\right]_{t-R_{t} / c_{0}},
$$

with $\mathrm{d} F=\ell\left(y_{1}, \theta, t\right) r_{0} \mathrm{~d} \theta \mathrm{d} y_{1}$. This expression must be integrated over the ring surface to provide the total acoustic pressure $p^{\prime}$, and the corresponding Fourier transform is expressed as

$$
\tilde{p}(\boldsymbol{x}, \omega)=\frac{-\mathrm{i} \omega}{4 \pi c_{0} S_{0}^{2}} \int_{0}^{2 \pi} \int_{-c / 2}^{c / 2}\left(x_{2} \sin \theta+x_{3} \cos \theta\right) \tilde{\ell}\left(y_{1}, \theta, \omega\right) \mathrm{e}^{\mathrm{i} k R_{t}} r_{0} \mathrm{~d} \theta \mathrm{d} y_{1},
$$

$\tilde{\ell}$ being the Fourier transform of $\ell$. In the acoustic calculations, the guided-wave effect due to multiple sound reflections on the inner walls of the ring is neglected. The approximation becomes exact only in the limit of vanishing ratio $c / r_{0}$ corresponding to an arbitrary large aspect ratio, and is expected accurate enough in the present application with the typical value $c / D=0.3$. Furthermore at low Mach numbers $\left(M^{2} \ll 1\right)$ the modified distance $S_{0}$ can be taken as equal to the geometrical distance $R$ without significant error. 
The incident turbulence responsible for the unsteady lift $\ell$ is assumed frozen during its convection time over the ring surface. It is expressed as a sum of spatial Fourier components or gusts with all possible spanwise aerodynamic wavenumbers. In the present cylindrical geometry, helical gusts with discrete azimuthal wavenumbers are taken to ensure the condition of $2 \pi$ periodicity in the $\theta$ direction. The streamwise aerodynamical wavenumber $k_{1}$ is imposed by the angular frequency of interest $\omega$, as $k_{1}=K_{1}=\omega / U$. Thus a relevant expression for $\tilde{\ell}$ is

$$
\tilde{\ell}\left(y_{1}, \theta, \omega\right)=2 \pi \rho_{0} \sum_{n=-\infty}^{\infty} \tilde{w}_{n}\left(K_{1}\right) g\left(y_{1}, K_{1}, \kappa_{n}\right) \mathrm{e}^{\mathrm{i} n \theta},
$$

where $g$ is a response function to be derived separately. Here $\kappa_{n}=n / r_{0}$ is the spanwise or azimuthal wavenumber related to the curvilinear coordinate $s$ and defined by $\kappa_{n} s=\kappa_{n} r_{0} \theta=n \theta . \tilde{w}_{n}\left(K_{1}\right)$ denotes the radial velocity amplitude of the gust of order $n$. Each gust forces a spinning pressure pattern on the ring with phase variations tuned by the exponential $\mathrm{e}^{\mathrm{i}(n \theta-\omega t)}$. It is equivalent to an oscillatory mode of the jet shear layer and the integer $n$ will be called the order of the gust or of the mode. A gust will be said subcritical or supercritical, as stated in Appendix, depending on either its azimuthal phase speed $\omega / n$ is subsonic or supersonic with respect to the moving fluid at speed $U$. The key point is the determination of $\tilde{\ell}$ or of the response function $g$ for any gust. In principle, this should be achieved by solving the linearized equations of gasdynamics in cylindrical coordinates. Instead of doing so and because the analysis addresses high frequencies, the unsteady response of the ring to a helical gust is assumed the same as the one of a thin rectangular plate to an oblique gust in Cartesian coordinates, for which different expressions are already available in the literature. This simplification is discussed in Appendix. More precisely, the present rectangular-aerofoil aerodynamic responses for arbitrary oblique gusts are a generalization of Amiet's original high-frequency solution for parallel gusts (Amiet 1976; Mish \& Devenport 2006). They are also reminded in Appendix. In principle, the high-frequency assumption holds for a non-compact chord $c$, thus typically a Helmholtz number $k c$ larger than 1 . Nevertheless the application will prove that it remains reliable at much lower values.

The power spectral density of the acoustic pressure in the far field is expressed as

$$
\begin{aligned}
S_{p p}(\boldsymbol{x}, \omega)= & \left(\frac{\omega}{4 \pi c_{0} S_{0}^{2}}\right)^{2} \iint_{0}^{2 \pi} \iint_{-c / 2}^{c / 2} S_{\ell \ell}\left(y_{1}, y_{1}^{\prime}, \theta, \theta^{\prime}, \omega\right) \mathrm{e}^{\mathrm{i} k\left(R_{t}-R_{t}^{\prime}\right)} \\
& \times\left(x_{2} \sin \theta+x_{3} \cos \theta\right)\left(x_{2} \sin \theta^{\prime}+x_{3} \cos \theta^{\prime}\right) r_{0}^{2} \mathrm{~d} \theta \mathrm{d} \theta^{\prime} \mathrm{d} y_{1} \mathrm{~d} y_{1}^{\prime},
\end{aligned}
$$

where $S_{\ell \ell}$ is the cross-spectral power density of the local unsteady lift, expressed as the ensemble average

$$
\lim _{T_{\infty} \rightarrow \infty}\left\{\frac{\pi}{T_{\infty}} \mathscr{E}\left[\tilde{\ell}\left(y_{1}, \theta, \omega\right) \tilde{\ell}^{*}\left(y_{1}^{\prime}, \theta^{\prime}, \omega\right)\right]\right\} .
$$

As pointed out by Amiet (1975), the incident turbulent field is assumed to extend over an arbitrary large domain from $-R_{\infty}$ to $R_{\infty}$ in the streamwise direction and corresponding time integrations are defined from $-T_{\infty}$ to $T_{\infty}$, with $R_{\infty} / T_{\infty}=U$. The random nature of the unsteady lift results from that of the oncoming velocity and the expected value must be displaced onto the product $\tilde{w}_{n} \tilde{w}_{m}^{*}$. This quantity can be shown to be related to the two-dimensional wavenumber spectrum of the turbulent velocity component normal to the ring surface $\Phi_{w w}$, accounting for the statistical 
orthogonality of the azimuthal wavenumbers, as

$$
\lim _{T_{\infty} \rightarrow \infty}\left\{\frac{\pi}{T_{\infty}} \mathscr{E}\left[\tilde{w}_{n}\left(K_{1}\right) \tilde{w}_{m}^{*}\left(K_{1}\right)\right]\right\}=2 \pi \frac{U}{r_{0}} \delta(n-m) \Phi_{w w}\left(K_{1}, \kappa_{n}\right) .
$$

In this expression $r_{0}$ is the scaling factor between the mode order $n$ and the corresponding wavenumber $\kappa_{n}$. $\Phi_{w w}$ will be given the same formal expression as the equivalent quantity for model turbulence in Cartesian coordinates. No specific development in terms of axisymmetric turbulence is attempted since the formalism is mainly dedicated to the assessment of Amiet's response function for oblique gusts. Finally,

$$
\begin{aligned}
S_{\ell \ell}\left(y_{1}, y_{1}^{\prime}, \theta, \theta^{\prime}, \omega\right) & =\left(2 \pi \rho_{0}\right)^{2} \frac{2 \pi U}{r_{0}} \\
& \times \sum_{n=-\infty}^{\infty} \Phi_{w w}\left(K_{1}, \kappa_{n}\right) \mathrm{e}^{\mathrm{i} n\left(\theta-\theta^{\prime}\right)} g\left(y_{1}, K_{1}, \kappa_{n}\right) g^{*}\left(y_{1}^{\prime}, K_{1}, \kappa_{n}\right) .
\end{aligned}
$$

The projected coordinates $(T, \Theta)$ of the observer in the plane $\left(x_{2}, x_{3}\right)$, such that $x_{2}=T \sin \Theta, x_{3}=T \cos \Theta$, are now used in the derivations (see figure 8), so that the difference in modified distance reads

$$
R_{t}-R_{t}^{\prime}=\frac{1}{\beta^{2} S_{0}}\left[\left(x_{1}-M S_{0}\right)\left(y_{1}^{\prime}-y_{1}\right)+\beta^{2} r_{0} T\left(\cos \left(\theta^{\prime}-\Theta\right)-\cos (\theta-\Theta)\right)\right] .
$$

Introducing this and the net result for $S_{\ell \ell}$ in the expression for the far-field sound yields

$$
S_{p p}(\boldsymbol{x}, \omega)=\left(\frac{k \rho_{0} c}{4 S_{0}^{2}}\right)^{2} 2 \pi r_{0} U \sum_{n=-\infty}^{\infty} \Phi_{w w}\left(K_{1}, \kappa_{n}\right)\left|\mathscr{L}\left(x_{1}, K_{1}, \kappa_{n}\right)\right|^{2}|\mathscr{I}|^{2},
$$

where $\mathscr{L}$ stands for the normalized aeroacoustic transfer function

$$
\mathscr{L}\left(x_{1}, K_{1}, \kappa_{n}\right)=\int_{-1}^{1} g\left(y_{1}^{*}, K_{1}, \kappa_{n}\right) \mathrm{e}^{-\mathrm{i}\left[k c\left(x_{1}-M S_{0}\right) y_{1}^{*}\right] /\left[2 \beta^{2} S_{0}\right]} \mathrm{d} y_{1}^{*},
$$

with $y_{1}^{*}=2 y_{1} / c$, given in the Appendix, and where $\mathscr{J}$ is the integral

$$
\mathscr{J}=\mathrm{e}^{\mathrm{i} n \Theta} \int_{0}^{2 \pi}[T \cos (\theta-\Theta)] \mathrm{e}^{-\mathrm{i} k r_{0} T \cos (\theta-\Theta) / S_{0}} \mathrm{e}^{\mathrm{i} n(\theta-\Theta)} \mathrm{d} \theta,
$$

readily put in the form i $T \mathrm{e}^{\mathrm{i} n \Theta} \partial \mathscr{I} / \partial a$ with (Abramowitz \& Stegun 1970)

$$
\mathscr{I}=\int_{0}^{2 \pi} \mathrm{e}^{-\mathrm{i} a \cos \xi} \mathrm{e}^{\mathrm{i} n \xi} \mathrm{d} \xi=2 \pi(-\mathrm{i})^{n} J_{n}(a), \quad a=\frac{k r_{0} T}{S_{0}} .
$$

Multiplying by the complex conjugate of $\mathscr{J}$, this yields the factor

$$
|\mathscr{F}|^{2}=(2 \pi)^{2} T^{2}\left(J_{n}^{\prime}\left(\frac{k r_{0} T}{S_{0}}\right)\right)^{2} .
$$

The final expression for the PSD of the acoustic pressure in the far field is

$$
\begin{aligned}
S_{p p}(\boldsymbol{x}, \omega)=\left(\frac{\pi k c \rho_{0}}{2 S_{0}^{2}}\right)^{2} 2 \pi r_{0} U \sum_{n=-\infty}^{\infty} & \Phi_{w w}\left(K_{1}, \kappa_{n}\right) \\
& \times\left|\mathscr{L}\left(x_{1}, K_{1}, \kappa_{n}\right)\right|^{2}\left(J_{n}^{\prime}\left(\frac{k r_{0} T}{S_{0}}\right)\right)^{2} .
\end{aligned}
$$


Equation (3.2) is the cylindrical equivalent of the formula derived by Amiet (1975) for a rectangular aerofoil, reproduced as (A 1) in Appendix. The squared derivative of the Bessel function is specific to the circular geometry and replaces the 'sinc' function typical of the radiation from sources distributed on a rectangle. Another difference is that the summation over all possible spanwise wavenumbers is discrete in (3.2) whereas it is continuous in Amiet's formulation. As expected, the angle $\Theta$ has no effect on the solution, since the sound field is statistically axisymmetric. Closed-form expressions for the function $\mathscr{L}$ are given in the Appendix.

\section{Application results and discussion}

\subsection{Input data}

Equation (3.2) is aimed at predicting the far-field broadband noise radiated from the ring but requires that the wavenumber velocity spectrum $\Phi_{w w}$ be known as input. This quantity is available at the price of dedicated experimental techniques, typically hot-wire measurements with at least a cross-wire probe in order to evaluate different components of the fluctuations and the anisotropy of the incident turbulence. From a practical point of view, the interest of the analytical approach is to provide fast prediction tools for manufacturers or end users who generally cannot afford to supply a model with very accurate data on the flow field in industrial context. Only a minimum information such as the turbulent kinetic energy and the dissipation rate deduced from Reynolds-averaged Navier-Stokes (RANS) computations may be available, together with a model of homogeneous and isotropic turbulence, assuming that the actual turbulent field is not far from the model one. For the sake of acoustic applications with an expected accuracy of a couple of decibels, the function $\Phi_{w w}$ can thus be given its closed-form expression according to the von Kármán or the Liepmann model. Useful definitions and formulae are found in the classical literature on turbulence (Hinze 1975) and are reminded for instance by Paterson \& Amiet (1976). The model is tuned by fitting the root mean square value $\sqrt{\bar{w}^{2}}=\sqrt{\overline{u^{2}}}$ and the integral length scale $\Lambda$ on the RANS data. In the present study, a more accurate description of the flow features is needed in principle. However the focus is rather limited to a minimum characterization of the turbulence properties involved in the aeroacoustic model. Therefore only the frequency spectrum $S_{u u}$ of the streamwise velocity component is measured. This is achieved on the set-up of figure $1(b)$ with a single-wire probe traversing the mixing layer of the jet, at the exact axial location to be given the ring leading edge but with the ring removed. Other related statistical quantities are deduced from complementary results reported in the literature. The assumption of equivalent homogeneous and isotropic turbulence made in the model is apparently questionable and crucial in a work made for validation purposes. It is acceptable if the statistical properties of the real flow involved in the aeroacoustic model are close to the corresponding properties in model turbulence. In the following the departures from isotopy and/or homogeneity are accounted for by corrections to be included in the model.

The anemometry results are reported in figure $9(a)$, for an axial distance $d=2 D$ and radial distances from the jet axis $r$ ranging from $2 r / D=0.8$ to $2 r / D=1.2$. This corresponds to values of the local mean flow velocity $U$ ranging from $31 \mathrm{~m} \mathrm{~s}^{-1}$ at the inner measuring point to $11 \mathrm{~m} \mathrm{~s}^{-1}$ at the outer one, for the exhaust flow speed $U_{0}=38 \mathrm{~m} \mathrm{~s}^{-1}$. In such conditions, the turbulence cannot be strictly homogeneous and a particular attention must be paid to the statistical properties of the flow in the vicinity of the ring location. Obviously, the spectral content increases in low 

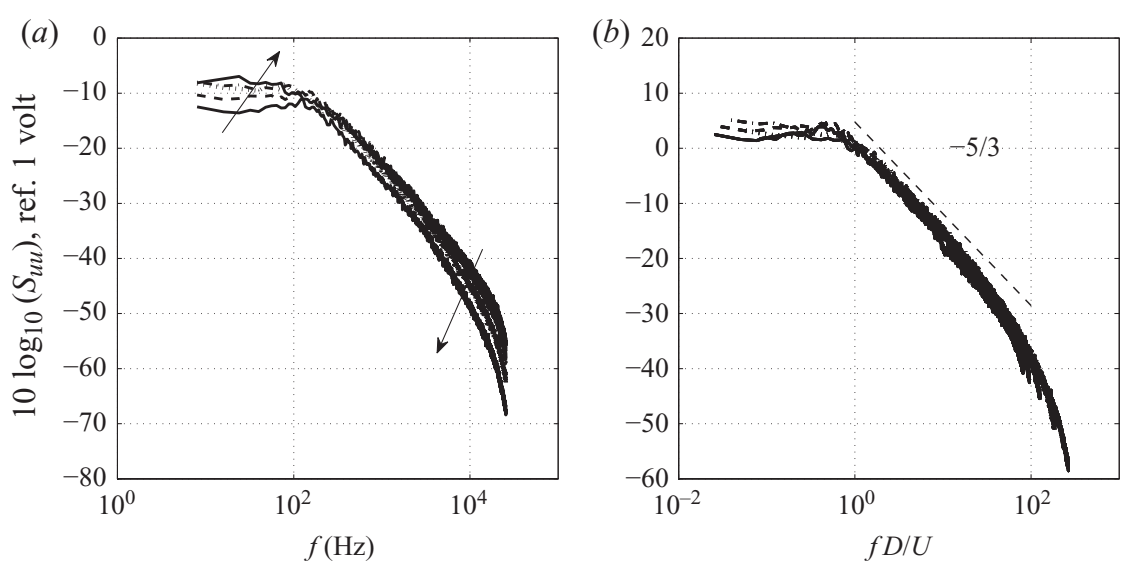

FIgURE 9. (a) Measured velocity spectra at different radii across the mixing layer and at a distance $d=2 D=20 \mathrm{~cm}$. Values of $2 r / D:(0.8,0.9,1,1.1,1.2)$, corresponding root mean square values of the axial fluctuations: $(5.66,6.49,6.73,6.14,5.7) \mathrm{m} \mathrm{s}^{-1}$. Arrows indicate increasing $r$. (b) Same data in reduced variables, featuring the theoretical $(-5 / 3)$ law.

frequencies and decreases in high frequencies as the probe is moved from the inner part to the outer part of the mixing layer. Next plotting the velocity spectrum $S_{u u}$ multiplied by the local mean flow speed $U$ as a function of the Strouhal number $f D / U$ based on that speed and on the jet diameter produces a good collapse of the data, as shown in figure $9(b)$. This suggests that the turbulent intensity only weakly depends on the local mean velocity around the centre of the mixing layer. More precisely, the root mean square of the axial velocity fluctuations takes the values $(5.66,6.49,6.73,6.14,5.7) \mathrm{m} \mathrm{s}^{-1}$ as $r$ goes from $0.8 D / 2$ to $1.2 D / 2$ by steps of $0.1 D / 2$ and has a maximum at the centre of the shear layer, $r=D / 2$. The characteristic scale $L_{0}$ defined by $L_{0}^{-1}=\partial \sqrt{\bar{u}^{2}} / \partial r \times\left(1 / \sqrt{\bar{u}^{2}}\right)$ is arbitrary large at this location, thus exceeds any integral scale of the turbulent field. This is a first indication of local homogeneity. Furthermore, the theoretical high-frequency slope with the $-5 / 3$ exponent according to the von Kármán model is featured on the figure.

The corresponding model spectra are superimposed on the measurements in figure 10, where the plots are shifted from each other by steps of $-5 \mathrm{~dB}$ for clarity. The integral length scale is tuned to $\Lambda=1.5 \mathrm{~cm}$, and a Gaussian correction is applied to reproduce the drop to the Kolmogorov scale at the highest frequencies, by just multiplying the model expressions by the factor $\mathrm{e}^{-(9 / 4)\left(f / f_{K}\right)^{2}}, f_{K}$ being a Kolmogorov frequency adjusted for the fit. A close look reveals that the spectrum measured at $r=D / 2$ is more accurately fitted by the model over an extended frequency range. In contrast spectra measured elsewhere depart from the models by amounts of up to $3 \mathrm{~dB}$, essentially because of a small hump around the cutoff frequency. Again the centre part of the mixing layer appears as closer to homogeneity.

The streamwise velocity spectra are next plotted in different reduced variables in figure 11(a) for comparison with the more extended data base reported by Bradshaw et al. (1964) in the case of a subsonic jet of Mach number 0.3 and Reynolds number $3.8 \times 10^{5}$. Both sets of results are obtained at two diameters downstream of the nozzle exit and at the same radial locations $2 r / D=0.8,1,1.2$. They are in a good agreement in the middle-and-high frequency range but a typical $3 \mathrm{~dB}$ higher fluctuating level is observed at low frequencies in the present experiment. This higher level makes the spectral shape in the shear-layer centreline closer to a model spectrum. The 


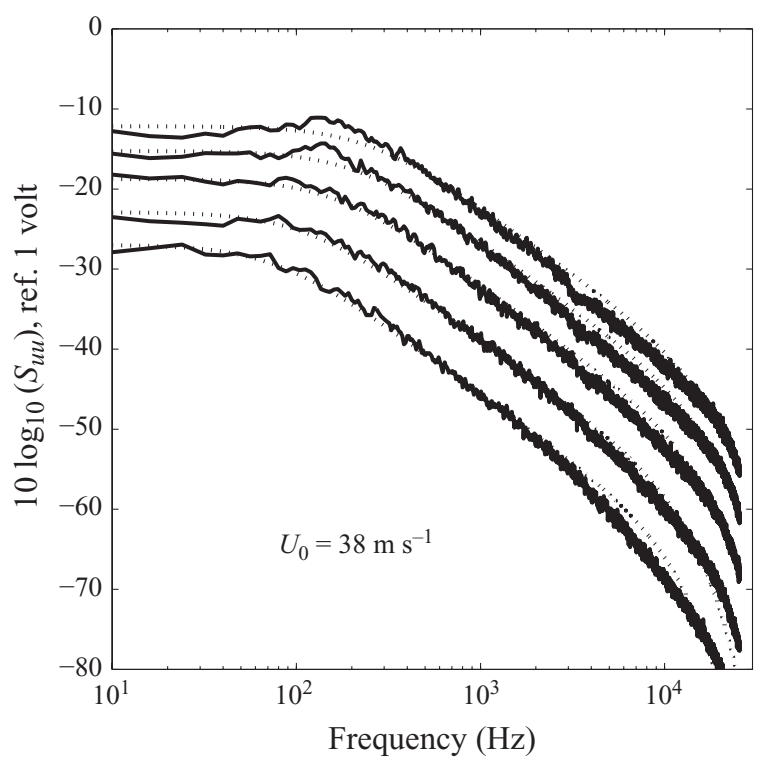

FiguRE 10. Same data as in figure 9 , vertically shifted by $-5 \mathrm{~dB}$ from each other, from $2 r / D=0.8$ (upper) to $2 r / D=1.2$ (lower). Theoretical fit according to the von Kármán model in dotted lines.
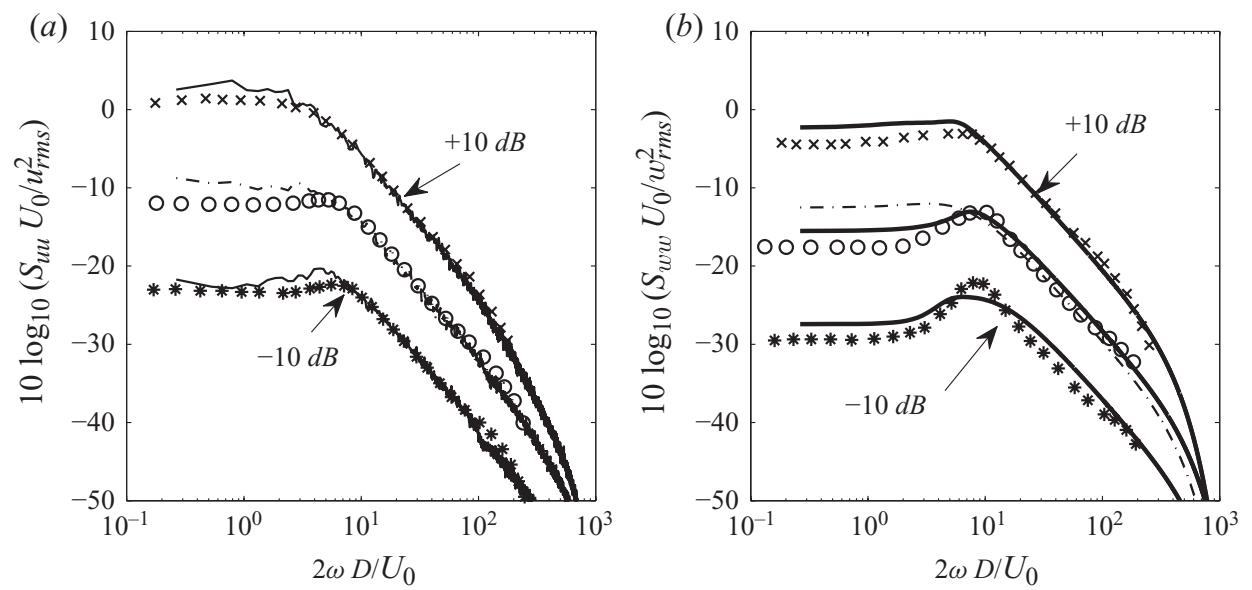

FigURE 11. (a) Normalized velocity spectra for the streamwise component from Bradshaw et al. (1964) (symbols), versus present measurements of figure 9 (lines). Data at different radii are shifted by $\pm 10 \mathrm{~dB}$ for clarity. $2 r / D=0.8\left(^{*}\right), 1(\mathrm{o})$ and $1.2(\times)$. (b) Reference normalized spectra for the radial component (symbols) and proposed modified homogeneous-turbulence spectra used for the acoustic calculations (thick lines). Original von Kármán spectrum on the centre layer in dashed-dotted line.

discrepancies between both experiments are attributed to a different development of the mixing layer because of the serrations. The overall qualitative agreement suggests that the missing spectra for the radial velocity component in the serratednozzle jet can be deduced from the normalized spectra measured by Bradshaw et al. (1964). The corresponding data are shown in figure 11(b). The spectrum on the centreline of the shear layer (symbol o) is first compared to a model von Kármán 
spectrum (dashed-dotted line). The agreement is good for reduced frequencies $4 \omega D / U_{0}$ ranging from 6 to 200, suggesting that the assumption of an equivalent homogeneous turbulence is relevant in the corresponding frequency range. However the fluctuations are clearly overestimated at low frequencies. This departure from ideal homogeneous and isotropic turbulence is prejudicial to the analytical aeroacoustic model and must be corrected. An ad hoc correction is provided by damping the low-frequency amplitude down to a value which remains $3 \mathrm{~dB}$ above the measured spectrum of Bradshaw et al. (1964) as in figure 11(a), and by adjusting the high-frequency drop. The resulting corrected spectrum to be used in the noise predictions is also plotted in figure 11(b), and similar corrections proposed for the other radial locations. It must be noted that the same low-frequency decrease and bump in the radial-velocity spectrum are reported by Ross et al. (2008) for the plane mixing layer.

Measurements performed on a high-speed subsonic jet and reported by Fleury et al. (2008) provide arguments according to which the turbulence in the shearlayer centreline is not isotropic. The reference experiment was made on a smaller jet (diameter of $5 \mathrm{~cm}$ ) at higher speeds (Mach numbers 0.6 and 0.9) thus the Reynolds numbers are sufficiently close to each other in both experiments for extending the observed trends to the present case (especially if the trends are not significantly Reynolds-dependent). The maximum root mean square values of the velocity fluctuations were found as $15 \%$ and $11 \%$ of the jet exit velocity for the streamwise and radial components, respectively. The data for the lateral mixing layer of a rectangular jet reported by Ross et al. (2008) exhibit the slightly higher values $18 \%$ and $12.5 \%$, respectively. These values give an indication of the amount of anisotropy in the flow. It is therefore relevant to consider that the streamwise fluctuating amplitude exceeds the radial one by a typical factor 1.4. For the sake of consistency this factor is applied later on when deducing the dimensional spectra used as input data in the aeroacoustic model. Additional statistical parameters also made available by Fleury et al. (2008) using Laser Doppler anemometry show that the turbulence in the centre part of the mixing layer exhibits some features of isotropic turbulence. First the contour plots of the space correlation functions were determined. The pattern of the function $R_{11}$ for the streamwise fluctuation was found slightly inclined in the plane of streamwise and radial separation variables $\left(\xi_{1}, \xi_{2}\right)$. In contrast the pattern for the function $R_{22}$ associated with the radial fluctuations was aligned with the axes. The length scales $L_{i i}^{(j)}$ with respect to $\xi_{j}$ were deduced by integration of the correlation functions $R_{i i}$. The ratios of correlation lengths $L_{11}^{(1)} / L_{22}^{(2)}$ and $L_{11}^{(2)} / L_{22}^{(1)}$ on the one hand, and $L_{11}^{(1)} / L_{11}^{(2)}$ and $L_{22}^{(2)} / L_{22}^{(1)}$ on the other hand, were found almost exactly equal to the theoretical values for isotropic turbulence, 1 and 2 respectively, for axial locations around two or three diameters downstream of the jet nozzle. Significant departures from isotropy were observed at larger downstream distances. This suggests that the turbulence would exhibit some isotropic features in the conditions of the present experimental configuration with a regular nozzle. The additional serrations are not expected to make any other difference than increasing the mixing process and possibly still enforce the isotropy.

As pointed out by Ross et al. (2008) for the plane shear layer, the departure from isotropy also affects the correlation length for low frequencies corresponding to values of the parameter $f \delta^{*} / U$ smaller than 0.1 , the actual length being larger than the model one. If the same is supposed to occur in the present experiment, discrepancies are expected below $300 \mathrm{~Hz}$. However, the azimuthal correlation length is probably reduced in the initial mixing region due to the serrations, as suggested by figure 2 . Furthermore the very low frequencies escape the range for which the aeroacoustic 

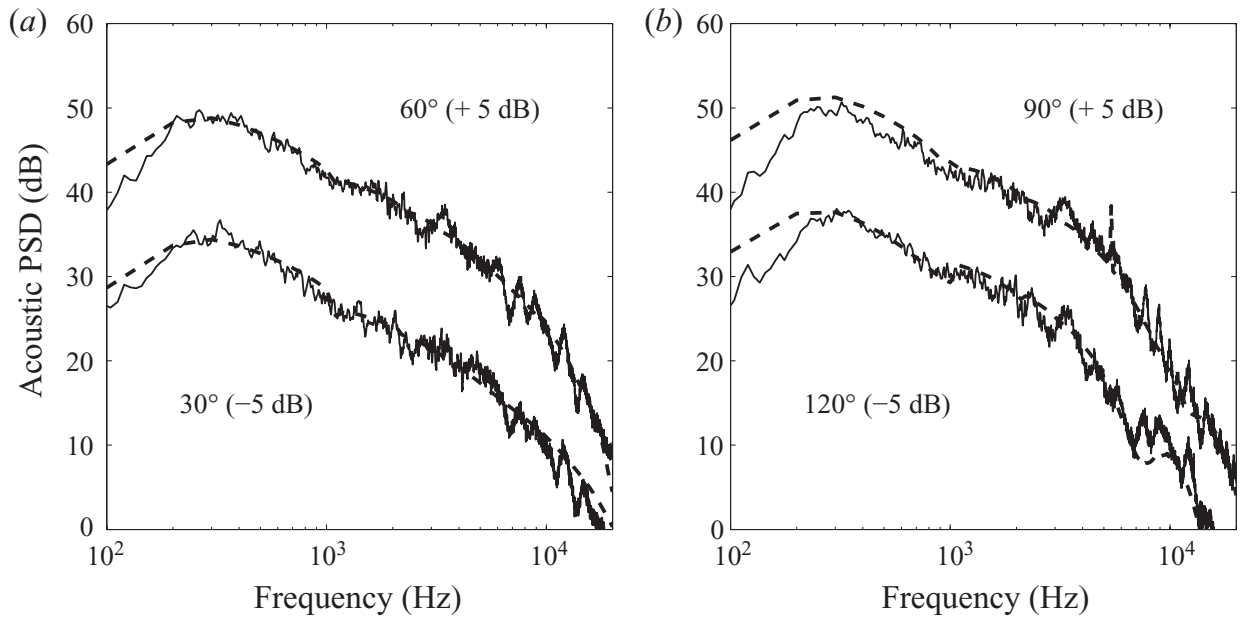

FIGURE 12. Theoretical far-field noise spectra (dashed lines) compared with the measurements (cont. lines). Input data from the point $2 r / D=1$ of figure 9. Reference ring of radius $r_{0}=D / 2$, interaction distance $d=2 D$, microphone distance $R_{m}=1.3 \mathrm{~m}$, jet exhaust velocity $U_{0}=38 \mathrm{~m} \mathrm{~s}^{-1}$. Radiation angles from the downstream jet axis $30^{\circ}, 60^{\circ}, 90^{\circ}, 120^{\circ}$. Couples of predicted and measured spectra shifted vertically for clarity.

response function is reliable. For these reasons, the model fit obtained here with an integral length scale $\Lambda=1.5 \mathrm{~cm}$ on the streamwise-velocity spectrum is retained for the intended acoustic purpose. The theoretical expression for the correlation length is taken as such and the aforementioned correction on the radial-velocity spectrum is applied. The frequency $300 \mathrm{~Hz}$ is considered the typical validity threshold of the analytical model with regard to the aerodynamic input data.

\subsection{Comparison of measured and predicted spectra}

Typical acoustic predictions are plotted in figure 12, for a jet exhaust velocity $U_{0}=38 \mathrm{~m} \mathrm{~s}^{-1}$ and the reference ring at a distance $d=20 \mathrm{~cm}=2 D$. The microphone is at a fixed distance $R_{m}=1.3 \mathrm{~m}$ and at different angles from the jet axis. The modes of orders ranging from -10 to +10 are taken into account, which is more than enough to ensure fully converged results over the investigated frequency range. Except for some over-prediction at the lowest frequencies, the agreement is all surprisingly good, since some questionable simplifications have been made in the analysis. Sound is first assumed to propagate in a medium uniformly moving at the local mean flow speed, here $U=23 \mathrm{~m} \mathrm{~s}^{-1}$ at the ring radial location, whereas the fluid is at rest outside the jet. Thus convective amplification effects are artificially introduced and the sound refraction by the jet shear layer profile is neglected. Corrections could be included for applications at higher Mach numbers but would make no sense in the present case. In fact, since at the low speeds investigated in the experiment the corrected distance $S_{0}$ is very close to the geometrical distance $R=\sqrt{x_{1}^{2}+x_{2}^{2}+x_{3}^{2}}$, the convective distortion is negligible. This is a general result for dipoles the axis of which is normal to the flow direction. The sound refraction cannot be simply included in the analysis but it is also expected to be weak. According to Amiet (1975) the solution based on the two-step Schwarzschild's technique holds for sufficiently high frequencies such that $k c /\left(2 \beta^{2}\right)>0.4$. The lower frequency limit of $300 \mathrm{~Hz}$ in the present case corresponds 


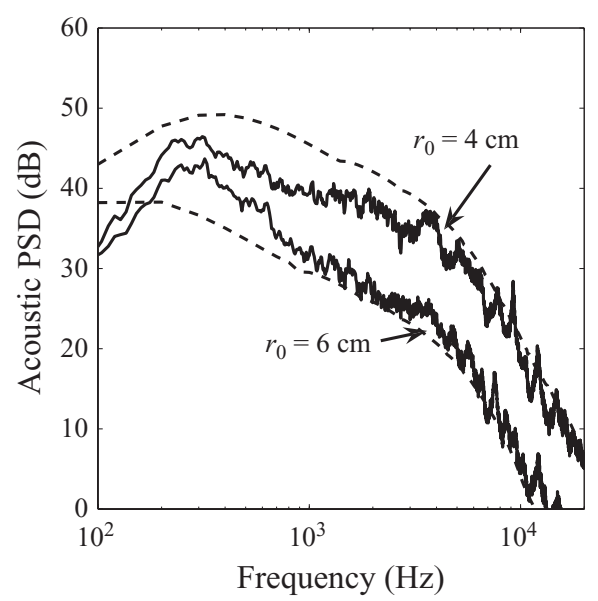

FIGURE 13. Theoretical far-field noise spectra compared with the measurements. Corrected input data from the points $2 r / D=0.8$ and $2 r / D=1.2$ of figure 9 . Rings of radii $r_{0}=0.4 \mathrm{~cm}$ and $r_{0}=0.6 \mathrm{~cm}$, interaction distance $d=2 D$, microphone distance $R_{m}=1.3 \mathrm{~m}$, jet exhaust velocity $U_{0}=38 \mathrm{~m} \mathrm{~s}^{-1}$. Radiation angle from the downstream jet axis $90^{\circ}$.

to $k c /\left(2 \beta^{2}\right) \simeq 0.12$ and the technique should be extended with further iterations. Such a refinement is not tractable analytically. Similar investigations of the trailing-edge noise of an aerofoil also based on Schwarzschild's technique suggest that the solution remains reliable for values of $k c /\left(2 \beta^{2}\right)$ lower than 0.25 (Roger \& Moreau 2005). Therefore the predictions at the lowest frequencies might be inaccurate because the model starts to deviate from the exact ring response function below, say $800 \mathrm{~Hz}$. In the absence of exact theoretical results for comparison, acceptable results are expected in terms of decibels at even lower frequencies remaining well above the $300 \mathrm{~Hz}$ threshold. For this reason, the present experimental results between, say $100 \mathrm{~Hz}$ and $500 \mathrm{~Hz}$ cannot be used to test the high-frequency response model because they are clearly out of its validity range. A possible extension of the study would be to derive alternative expressions based on a low-frequency analysis. This is out of the scope of the paper.

Further results obtained with the smaller and larger rings of diameters $2 r_{0}=0.8 D$ and $2 r_{0}=1.2 D$ are reported in figure 13 . The agreement is poor in these cases when the model is assessed against the measurements, even though the same procedure including the corrections for the radial-velocity spectra is applied. The sound level is underpredicted for the larger ring and overpredicted for the smaller one. Yet the slope modification in the spectral shape at high frequencies is reproduced correctly. The discrepancies occur over the low-and-middle frequency range, irrespective of the aforementioned limitation of the analytical model. The inaccuracy of the predictions is possibly explained by the non-homogeneity of the developing jet flow. Along the centre sheet of the mixing layer of radius $D / 2$, the mean-flow speed is nearly constant as shown in figure 4 . Thus as the ring of diameter $D$ is placed facing the nozzle, it encounters homogeneous conditions and the model assumptions are reasonable. In contrast a cylindrical cut in the mixing layer at other diameters corresponds to mean-flow parameters which significantly vary with the axial location (see the difference in figure 4 between the profiles at $d=2 D$ and $d=3 D$ ). The mean flow slows down axially in the inner region of the mixing layer and accelerates in the outer region. This variation may not be negligible along the chord length $c$. It 
makes the chordwise averaged relative flow over the ring of diameter $2 r_{0}=0.8 D$ smaller than a reference value taken at the ring leading edge. In the same way the averaged value for the ring of diameter $2 r_{0}=1.2 D$ is larger than the reference value. Thus calculations using values referenced at the leading edge as input data are somewhat expected to produce the aforementioned wrong estimates. No correction has been introduced, essentially because the effect of the non-homogeneity depends on frequency. Indeed the lift fluctuations induced by turbulence on an aerofoil are known to progressively concentrate towards the leading-edge as frequency increases. Another source of discrepancies could be due to the intermittency of the turbulent flow at the edges of the mixing layer.

The present comparison of measurements and predictions with the reference ring validates the use of the transposed Amiet's theory for ring noise calculations, except at low frequencies. As a consequence the response functions of a rectangular thin aerofoil to oblique, either subcritical or supercritical gusts are also validated. Oblique radiation from a thin aerofoil is preferentially forced by incident aerodynamic gusts the wavefronts of which make a finite angle with the spanwise direction (Amiet 1975). Such oblique gusts correspond to the helical gusts (or higher-order modes) in the present analysis. In contrast, sound radiation in the mid-span plane of a rectangular aerofoil is rather selected by gust wavefronts parallel to the span, which correspond to the symmetric mode in cylindrical coordinates.

\subsection{Interpretation of results}

A numerical splitting of the full analytical solution into modes or sets of modes is shown in figure 14, for two radiation angles and the same input data as for figure 12, in order to get more information on the dominant modes (or corresponding gusts). The contributions of the subcritical and supercritical gusts are featured separately, in thick continuous line and in dotted line respectively. The supercritical gusts alone are found to determine the sound field in the middle-and-high frequency range, whereas the subcritical gusts determine the low-frequency high-level part of the spectrum. Both curves cross each other at a point around $1 \mathrm{kHz}$ where the solution has a small jump. Additional jumps or discontinuities are observed on the subcritical solution set at higher frequencies, with no effect on the total result. The jumps occur at transition frequencies for which a given isolated mode turns from the subcritical to the supercritical regime. At such a frequency, the Schwarzschild's technique used in Amiet's solving procedure breaks down and a regularization should be applied to match continuously the solutions calculated on each side. A possible smoothening technique has been described and applied by Roger \& Moreau (2005) in a similar study, dealing with a three-dimensional model of the trailing-edge noise of an aerofoil. No equivalent attempt is made in the present paper, since the full solution remains satisfactory apart from the residual jump around $1 \mathrm{kHz}$. The latter is responsible for only a possible local error of a couple of decibels, depending on the radiation angle for which the calculations are performed. It is remarkable that, despite the multiple jumps, the subcritical gusts improve the regularity of the full solution where they give a significant contribution, as shown particularly in figure 14 for the angle of $120^{\circ}$ around $10 \mathrm{kHz}$ : the physically consistent hump in the full solution, mainly reproduced by the supercritical gusts and due to chordwise non-compactness, is smoothed.

The very significant contribution of the subcritical gusts in the circular geometry is a major result of the present study. In contrast, the subcritical gusts interacting with a rectangular plate in Cartesian coordinates would be much less efficient than the supercritical gusts, as pointed out by Graham (1970). In principle, the potential 

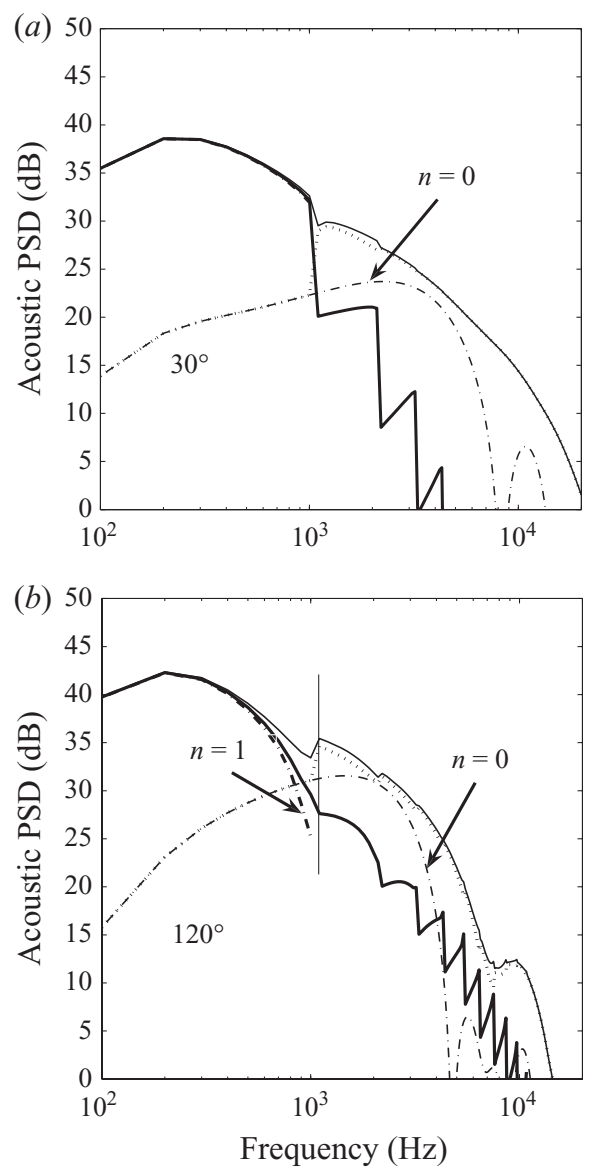

FIGURE 14. Gust breakdown of the predictions of figure 12 at $30^{\circ}$ and $120^{\circ}$, emphasizing the relative contributions of the subcritical (thick continuous line) and supercritical (dotted line) sets of gusts. First transition occurs at the vertical cut. Thin continuous line: total field. Calculations with the isolated modes $n=0$ or $n= \pm 1$ (dashed-dotted lines) are pointed by arrows.

induced by these gusts with subsonic azimuthal phase speeds is solution of an elliptic equation (Appendix). As such, it would not produce any sound in the far field in the limit of infinite span or aspect ratio. Their non-zero radiation for a rectangular plate only results from the finiteness of the span. Not only the subcritical helical gusts are clearly responsible for the low-frequency high-level bump below $1 \mathrm{kHz}$ in the noise spectrum within the range of parameters investigated, but more precisely, the bump is essentially produced by the first non-symmetric mode $n= \pm 1$. This is emphasized when the predictions are made with this mode only, and plotted as the black dashed line of figure 14(b). The mode $n= \pm 1$ is definitely the most efficient one in the subcritical range. This result is expected from the asymptotic behaviour of the Bessel function for small arguments. In the limit of very low frequencies for which $k r_{0} \ll 1$, only the function $J_{1}$ contributes because of its non vanishing derivative. The same mode has no trace above $1 \mathrm{kHz}$ where it experiences transition, suddenly contributing the set of the supercritical gusts. It is worth noting that the symmetric mode $n=0$ featured by the thin dashed-dotted line on the figure cannot be in the subcritical range. It is always supercritical since its angular phase speed is infinite. 
The symmetric mode becomes dominant only in the middle frequency range, above $2 \mathrm{kHz}$, but the higher-order modes contribute as well. The experimental evidence of the dominant role of the azimuthal modes of orders 0 and \pm 1 in the dynamics of subsonic free jets has been already reported by many authors (e.g. Lau \& Fisher 1975; Fuchs \& Michel 1978). It is confirmed indirectly in the present study through the acoustic signature of the modes, by both the analysis and the measurements.

The different efficiencies of the modes 0 and \pm 1 can be intuitively understood as follows. The varicose mode $n=0$ impinging on the ring generates equivalent distributed dipoles which are perfectly in phase all along the perimeter in the sense of the cylindrical coordinates, both the excitation and the response being axisymmetric. Two point dipoles, diametrically opposite, radiate in phase opposition with respect to a given direction of propagation taken in the same plane. Thus the signals they produce at a far-field observer tend to cancel each other at low frequencies, for which the differences in propagation time, related to the differences in the source-to-observer distance, remain small when compared to the period of oscillation. The lower the frequency is, the better the cancellation. As a result the couple of dipoles acts as a less efficient quadruple at frequencies for which the ring diameter is much smaller than the wavelength. Then the diameter is said acoustically compact. In contrast the mode $n= \pm 1$ induces equivalent dipoles which oscillate in phase opposition on two points diametrically opposite on the ring, again from the point of view of the cylindrical coordinates. But with respect to a given direction of propagation in the plane of the diameter, the two dipoles oscillate in phase. The net result at very low frequencies is a dipole of doubled strength since no cancellation can be expected from the too small differences of retarded time or of propagation distance. This explains why the contribution of the mode $n= \pm 1$ can exceed the one of the mode $n=0$ by typically $20 \mathrm{~dB}$ at low frequencies on the plots of figure 14. As frequency increases, less and less cancellation occurs between two diametrically opposite dipoles forced by the symmetric mode due to the increase of retarded time differences. Therefore the symmetric mode becomes more efficient. The frequency of its maximum efficiency depends on the direction of propagation since the retarded-time differences do. It is around 2 or $3 \mathrm{kHz}$ in figure 14. At even higher frequencies for which the ring is not compact anymore, and again for the symmetric mode, the retarded time differences along a diameter can result in the exact cancellation of the two interfering sounds received by the observer. Though the analysis is to be completed by all other couples of dipoles for which the observer is not in the plane of the diameter, the modal extinction is an expected feature. It occurs in figure 14 around $8500 \mathrm{~Hz}$ at $30^{\circ}$ and $5000 \mathrm{~Hz}$ at $120^{\circ}$. In the same way, increasing the frequency up to the first transition for the first azimuthal mode progressively reduces its efficiency by introducing cancellation between the sounds coming from diametrically opposite dipoles.

\section{Conclusions}

A circular jet-ring configuration has been investigated in the paper as an alternative to rectangular configurations addressing the turbulence-interaction noise of an aerofoil. The self-sustained oscillations excited for small jet-ring distances and a regular nozzle lip have been avoided by increasing the jet-ring distance and by using a serrated nozzle. Pure broadband noise is generated this way, the ring scattering the natural jet turbulence as sound. The partially measured statistical properties of the turbulence have been found in agreement with previously reported works and the missing quantities have been inferred from these works by rescaling non-dimensional data. 
A statistical analytical prediction model based on the acoustic analogy has been derived, involving the splitting of the incident turbulence into helical gusts. The model is dedicated to high frequencies, for which the local unsteady aerodynamic response of the ring in the streamwise direction can be assumed identical to the one for a rectangular aerofoil in Cartesian coordinates. The radial-velocity spectrum and the associated azimuthal correlation length are used as input data. Since the model is initially derived assuming homogeneous and isotropic turbulence, a correction is applied to account for the anisotropy found in the mixing layer of a jet. The literature on jet turbulence suggests that the centre part of the mixing layer, in the continuation of the nozzle lip, exhibits some properties that are also found in homogeneous and isotropic turbulence. These properties are believed to make the proposed correction reliable but they are not satisfied when approaching the inner and outer boundaries of the mixing layer. For this reason the far-field noise predictions are found in excellent agreement with the acoustic measurements when the ring has the same diameter as the nozzle, except at the lowest frequencies for which the model assumptions do not hold anymore. In contrast, the prediction methodology with a smaller and a larger ring failed.

For the Mach numbers and Reynolds numbers covered in the study, the subcritical gusts, with subsonic azimuthal phase speeds, are responsible for a low-frequency high-level bump in the acoustic spectrum, for which the ring is acoustically compact. The supercritical (supersonically spinning) patterns contribute to the middle-and-high frequency range, for which the ring is not compact anymore. By the way, important conclusions are drawn from the good agreement found between the predictions and the measurements in the present ring geometry. Firstly, the results validate the highfrequency three-dimensional transfer functions, both supercritical and subcritical, derived analytically. Secondly, they suggest that the subcritical gusts would play an important role in other configurations as well, typically for a curved surface and/or when considering sound radiation in directions which are not perpendicular to the surface. They would contribute to the radiation off the mid-span plane of a rectangular aerofoil, for instance, and to the free-field radiation from a fan with swept and leant blades. To the author's knowledge, this has not been pointed out clearly before, nor confirmed by experimental evidence.

The author is grateful to Stella Serafini (Politecnico di Torino, Italy) for her precious help in the experiment and her interest for the study of the self-sustained oscillations.

\section{Appendix. Subcritical and supercritical aeroacoustic response functions of a rectangular aerofoil}

The aeroacoustic response or transfer function $\mathscr{L}$ of the ring to incident helical gusts is now to be derived to complete the analysis of $\S 3$, from the expression of the unsteady lift $\tilde{\ell}$. For that goal, the additional disturbance introduced by the ring into the turbulent flow is described at the angular frequency $\omega$ by a potential $\phi \mathrm{e}^{-\mathrm{i} \omega t}$. This potential is solution of the linearized equations of compressible non-dissipative fluid dynamics, eventually recast as the convected Helmholtz equation

$$
\frac{\partial^{2} \phi}{\partial r^{2}}+\frac{1}{r} \frac{\partial \phi}{\partial r}+\frac{1}{r^{2}} \frac{\partial^{2} \phi}{\partial \theta^{2}}+\beta^{2} \frac{\partial^{2} \phi}{\partial z^{2}}+2 \mathrm{i} k M \frac{\partial \phi}{\partial z}+k^{2} \phi=0
$$

in cylindrical coordinates. Once determined, $\phi$ provides the expressions of the disturbance pressure, and the induced lift is defined as the pressure jump across the surface. Solving the equation directly in the reference frame of figure 8 is not 
attempted in the paper, for the sake of simplicity on the one hand, and precisely because the aim is to assess the validity of assimilating the exact response to the model response of a rectangular aerofoil, on the other hand. Without going into deeper arguments, the simplification is partly justified as follows. Under the assumption of small incident disturbances, the unsteady aerodynamic phenomena leading to the onset of lift fluctuations concentrate in an annular slice of fluid of mean radius $r_{0}$ close to the ring surface. Let $h$ be the characteristic radial thickness of this slice. In the non-dimensional variables $X=\left(r-r_{0}\right) / h, Y=r \theta / h, Z=z / h$ introduced to assess the limit for small values of $h / r_{0}$, the Helmholtz equation reads

$$
\frac{\partial^{2} \phi}{\partial X^{2}}+\frac{h}{r_{0}} \frac{\partial \phi}{\partial X}+\frac{\partial^{2} \phi}{\partial Y^{2}}+\beta^{2} \frac{\partial^{2} \phi}{\partial Z^{2}}+2 \mathrm{i}\left(k r_{0}\right) \frac{h}{r_{0}} M \frac{\partial \phi}{\partial Z}+\left[\left(k r_{0}\right) \frac{h}{r_{0}}\right]^{2} \phi=0
$$

Therefore, at high frequencies for which the parameter $k r_{0}$ is large enough for $k h$ to be of order 1 , the second term of the differential operator can be discarded and the equation reduces to the Helmholtz equation in Cartesian coordinates, which is the background of most linearized thin-aerofoil theories (Graham 1970). In other words, the unsteady response of the ring to a helical gust can be evaluated by solving the simpler problem of the response of a thin rectangular plate to an oblique gust in Cartesian coordinates. This high-frequency approximation a priori holds above $1 \mathrm{kHz}$ in the present study. If proved reliable by comparison with measurements, it provides an indirect way to validate the response functions of a rectangular aerofoil for radiation off the mid-span plane. Now the compressible, unsteady aerodynamic response of a thin rigid plate of infinite span to turbulence has been investigated mainly in the seventies (e.g. Graham 1970; Amiet 1975, 1976). According to the linearized theory, based on a Fourier decomposition of the turbulent field in sinusoidal gusts, only the velocity fluctuations normal to the plate surface contribute to the unsteady lift. The latter depends on the three parameters of a gust, namely the convective Mach number $M$ and the two aerodynamic wavenumbers $k_{1}$ and $k_{2}$ in the chordwise and spanwise directions, respectively. Apart from other pioneer works not cited here for conciseness, Amiet (1975) derived a closed-form high-frequency solution for compressible parallel gusts $\left(k_{2}=0\right)$ by means of an elegant application of the Schwarzschild's procedure also discussed by Landahl (1961). Other solutions were provided, for instance, by Filotas (1969) for oblique incompressible gusts $(M=0)$. In principle, the general solution can be obtained from the aforementioned ones using similarity rules derived by Graham (1970). Alternatively, the problem of the threedimensional compressible gust can be solved directly by applying Schwarzschild's theorem to a transformed set of variables and equations (Amiet 1976). Finally, the induced lift is used to evaluate the far-field pressure by calculating a radiation integral. The main steps of the analysis are rapidly outlined in this Appendix since they are nothing but straightforward extensions of Amiet's original work, and the reader is invited to go to the reference paper for more details (Amiet 1976). A Cartesian coordinate system is attached to the rectangular plate as shown in figure 15 . The source coordinates $\left(y_{1}, y_{2}, y_{3}\right)$ in the streamwise, spanwise and normal directions respectively are made non-dimensional by the half chord length:

$$
y_{1}^{*}=2 \frac{y_{1}}{c}, \quad y_{2}^{*}=2 \frac{y_{2}}{c}, \quad y_{3}^{*}=2 \beta \frac{y_{3}}{c} .
$$

The observer coordinates are noted $\boldsymbol{x}=\left(x_{1}, x_{2}, x_{3}\right)$.

Expressions of the induced fluctuating lift or local pressure jump across the plate are given, for instance, by Mish \& Devenport (2006), following Amiet (1976). When the phase speed of the trace of a gust on the plate leading edge, with respect to 


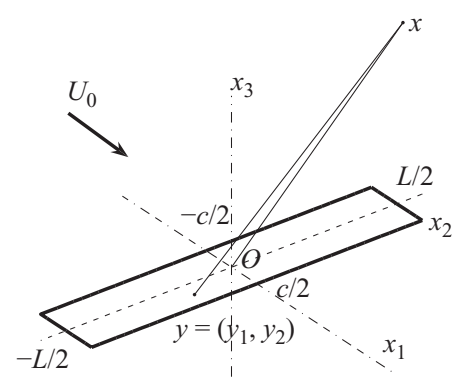

FIGURE 15. Reference frame centred on a rectangular aerofoil of aspect ratio $L / c$.

the moving fluid, is supersonic, the gust is said supercritical. In this case Graham's parameter $\Theta_{0}=k_{1}^{*} M /\left(\beta k_{2}^{*}\right)$ is larger than 1 . The complementary gusts for which $\Theta_{0}<1$ and the trace speed is subsonic are said subcritical. The local induced unsteady lift can be written as $\tilde{\ell}^{A}=\tilde{\ell}_{1}^{A}+\tilde{\ell}_{2}^{A}$ where the subscripts $(1,2)$ stand for the dominant leading-edge scattering and for the Kutta-condition correction, respectively. For the subcritical gusts, the result is

$$
\begin{aligned}
& \tilde{\ell}_{1}^{A}\left(y_{1}^{*}, y_{2}^{*}, \omega\right)=\frac{-2 \rho_{0} U_{0} w_{0} \mathrm{e}^{\mathrm{i} \pi / 4}}{\sqrt{\pi\left(k_{1}^{*}+\mathrm{i} \beta^{2} \bar{\kappa}^{\prime}\right)\left(1+y_{1}^{*}\right)}} \mathrm{e}^{-\left(\bar{\kappa}^{\prime}+\mathrm{i} M \bar{\mu}\right)\left(1+y_{1}^{*}\right)} \mathrm{e}^{\mathrm{i} k_{2}^{*} y_{2}^{*}}, \\
& \tilde{\ell}_{2}^{A}\left(y_{1}^{*}, y_{2}^{*}, \omega\right)=\frac{2 \rho_{0} U_{0} w_{0} \mathrm{e}^{\mathrm{i} \pi / 4}}{\sqrt{2 \pi\left(k_{1}^{*}+\mathrm{i} \beta^{2} \bar{\kappa}^{\prime}\right)}} \mathrm{e}^{-\left(\bar{\kappa}^{\prime}+\mathrm{i} M \bar{\mu}\right)\left(1+y_{1}^{*}\right)} \mathrm{e}^{\mathrm{i} k_{2}^{*} y_{2}^{*}}\left\{1-\operatorname{erf}\left(\sqrt{2 \bar{\kappa}^{\prime}\left(1-y_{1}^{*}\right)}\right)\right\},
\end{aligned}
$$

where erf is the ordinary error function, and

$$
\bar{\kappa}^{\prime}=\sqrt{\frac{k_{2}^{* 2}}{\beta^{2}}-\bar{\mu}^{2}}, \quad \bar{\kappa}^{2}=\bar{\mu}^{2}\left(\frac{1}{\Theta_{0}^{2}}-1\right), \quad \bar{\mu}=\frac{k c}{2 \beta^{2}}=\frac{k_{1}^{*} M}{\beta^{2}}, \quad k_{j}^{*}=\frac{k_{j} c}{2} .
$$

The last step is the calculation of the radiated far field according to the acoustic analogy (Ffowcs Williams \& Hawkings 1969), in which the lift stands for the equivalent source distribution, using the same background as in $\S 3$. In this calculation propagation path differences from the source points towards the observer are introduced. It must be noted that, if determined in whole space, the potential disturbance field $\phi$ would provide the acoustic field. However, calculating the induced lift or the trace of the potential on the plate surface is a more tractable task, achieved assuming that the span is of infinite extent to get rid of end effects. The acoustic radiation is evaluated in a second step from the induced lift by integrating over the actual span. This simplification leads to closed-form expressions at all steps of the analysis. The power spectral density of the far-field acoustic pressure is formally obtained as

$$
\begin{aligned}
S_{p p}(x, \omega)=\left(\frac{k \rho_{0} c x_{3}}{2 S_{0}^{2}}\right)^{2} \pi U_{0} \frac{L}{2} \int_{-\infty}^{\infty} \Phi_{w w}\left(\frac{\omega}{U_{0}}, k_{2}\right) \\
\times\left|\mathscr{L}\left(x_{1}, \frac{\omega}{U_{0}}, k_{2}\right)\right|^{2} \frac{\sin ^{2}\left[\left(\frac{k x_{2}}{S_{0}}-k_{2}\right) \frac{L}{2}\right]}{\pi \frac{L}{2}\left(\frac{k x_{2}}{S_{0}}-k_{2}\right)^{2}} \mathrm{~d} k_{2},
\end{aligned}
$$


where $\mathscr{L}$ is the non-dimensional chordwise aeroacoustic transfer function

$$
\mathscr{L}\left(x_{1}, \frac{\omega}{U_{0}}, k_{2}\right)=\int_{-1}^{1} \frac{\tilde{\ell}^{A} \mathrm{e}^{-\mathrm{i} k_{2}^{*} y_{2}^{*}}}{2 \pi \rho_{0} w_{0}} \mathrm{e}^{-\mathrm{i} \bar{\mu}\left(\frac{x_{1}}{S_{0}}-M\right) y_{1}^{*}} \mathrm{~d} y_{1}^{*} .
$$

This transfer function is found the sum of two contributions related to $\tilde{\ell}_{1}^{A}$ and $\tilde{\ell}_{2}^{A}$, as $\mathscr{L}=\mathscr{L}_{1}+\mathscr{L}_{2}$ with the expressions

$$
\begin{gathered}
\mathscr{L}_{1}=-\frac{1}{\pi} \sqrt{\frac{2}{\left(K_{1}^{*}+\mathrm{i} \beta^{2} \bar{\kappa}^{\prime}\right)\left(\mathrm{i} \bar{\kappa}^{\prime}-\bar{\mu} x_{1} / S_{0}\right)}} \mathrm{e}^{-\mathrm{i} \Theta_{2}} E\left[2\left(\mathrm{i} \bar{\kappa}^{\prime}-\bar{\mu} \frac{x_{1}}{S_{0}}\right)\right], \\
\mathscr{L}_{2}=\frac{\mathrm{e}^{-\mathrm{i} \Theta_{2}}}{\pi \sqrt{2 \pi\left(K_{1}^{*}+\mathrm{i} \beta^{2} \bar{\kappa}^{\prime}\right)} \Theta_{3}}\left\{1-\mathrm{e}^{-2 \Theta_{3}}-\operatorname{erf}\left(\sqrt{4 \bar{\kappa}^{\prime}}\right)\right. \\
\left.+2 \mathrm{e}^{-2 \Theta_{3}} \sqrt{\frac{\bar{\kappa}^{\prime}}{\mathrm{i} \bar{\kappa}^{\prime}+\bar{\mu} x_{1} / S_{0}}} E\left[2\left(\mathrm{i} \bar{\kappa}^{\prime}+\bar{\mu} x_{1} / S_{0}\right)\right]\right\},
\end{gathered}
$$

where

$$
\Theta_{3}=\bar{\kappa}^{\prime}+\mathrm{i} \bar{\mu} \frac{x_{1}}{S_{0}}, \quad \Theta_{2}=\bar{\mu}\left(M-x_{1} / S_{0}\right)-\pi / 4 .
$$

In the formulae, the complex error function $U^{0}(Z)$ is used to extend the Fresnelintegral function $E$ introduced by Amiet to complex arguments:

$$
U^{0}(Z)=\frac{1}{\sqrt{\pi}} \int_{0}^{Z^{2}} \frac{\mathrm{e}^{-z}}{\sqrt{z}} \mathrm{~d} z=(1-\mathrm{i}) E\left(\mathrm{i} Z^{2}\right), \quad E(\xi)=\int_{0}^{\xi} \frac{\mathrm{e}^{\mathrm{i} t}}{\sqrt{2 \pi t}} \mathrm{~d} t .
$$

The alternative expressions for a supercritical oblique, compressible gust $\left(\Theta_{0}>1\right.$ and $\left.\bar{\kappa}^{\prime 2}<0\right)$ are deduced from the preceding ones by simply changing i $\bar{\kappa}^{\prime}$ in $\bar{\kappa}=\left[\bar{\mu}^{2}-\right.$ $\left.\left(k_{2}^{2} / \beta\right)^{2}\right]^{1 / 2}$ and by replacing the error function $\operatorname{erf}(\sqrt{\cdot})$ by the function $(1-i) E$. This yields

$$
\begin{gathered}
\mathscr{L}_{1}=-\frac{1}{\pi} \sqrt{\frac{2}{\left(K_{1}^{*}+\beta^{2} \bar{\kappa}\right) \Theta_{4}}} \mathrm{e}^{-\mathrm{i} \Theta_{2}} E\left[2 \Theta_{4}\right], \\
\mathscr{L}_{2}=\frac{\mathrm{e}^{-\mathrm{i} \Theta_{2}}}{\pi \Theta_{4} \sqrt{2 \pi\left(K_{1}^{*}+\beta^{2} \bar{\kappa}\right)}}\left\{\mathrm{i}\left(1-\mathrm{e}^{2 \mathrm{i} \Theta_{4}}\right)-(1+\mathrm{i})\right. \\
\left.\times\left[E(4 \bar{\kappa})-\mathrm{e}^{2 \mathrm{i} \Theta_{4}} \sqrt{\frac{2 \bar{\kappa}}{\bar{\kappa}+\bar{\mu} x_{1} / S_{0}}} E\left[2\left(\bar{\kappa}+\bar{\mu} x_{1} / S_{0}\right)\right]\right]\right\}
\end{gathered}
$$

with

$$
\Theta_{4}=\bar{\kappa}-\bar{\mu} \frac{x_{1}}{S_{0}} .
$$

Supercritical gusts would be the only radiating patterns for a rectangular plate in the limit of infinite span (large aspect ratio approximation). However, the subcritical gusts contribute as well in the case of a finite span, as already pointed out in a similar formulation for the trailing-edge noise of an aerofoil by Roger \& Moreau (2005).

The preceding results are finally transposed to helical gusts in cylindrical geometry. For this the spanwise coordinate $y_{2}$ is just replaced by the curvilinear coordinate in the tangential direction, and a periodicity condition is applied as explained in the main text. The solution for the supercritical regime is given by expressions (A 4) and (A 5) in which $\kappa$ is replaced by the parameter $\bar{\kappa}_{n}$ defined from $\kappa_{n}$ instead of $k_{2}$. 
In the case of the subcritical gusts, (A 2) and (A 3) hold with $\bar{\kappa}_{n}^{\prime}=\left[\left(\kappa_{n} / \beta\right)^{2}-\bar{\mu}^{2}\right]^{1 / 2}$ instead of $\bar{\kappa}^{\prime}$. The modified versions of (A 2), (A 3), (A 4) and (A 5) are used as such in the analytical predictions of $\S 4$.

\section{REFERENCES}

Abramowitz, M. \& Stegun, I. A. 1970 Handbook of Mathematical Functions. Dover.

Amiet, R. K. 1975 Acoustic radiation from an airfoil in a turbulent stream. J. Sound Vib. 41 (4), 407-420.

Amiet, R. K. 1976 High frequency thin-airfoil theory for subsonic flow. AIAA J. 14 (8), 1076-1082.

Bradshaw, P., Ferris, D. H. \& Johnson, R. F. 1964 Turbulence in the noise-producing region of a circular jet. J. Fluid Mech. 19 (4), 591-624.

Brooks, T. F. \& Humphreys, W. M., JR. 2003 Flap-edge aeroacoustic measurements and predictions. J. Sound Vib. 261 (1), 31-74.

Curle, N. 1955 The influence of solid boundaries upon aerodynamic sound. Proc. R. Soc. A 231, 505-514.

Ffowcs Williams, J. E. \& Hawkings, D. L. 1969 Sound generation by turbulence and surfaces in arbitrary motion. Proc. R. Soc. 264, 321-342.

Filotas, L. T. 1969 Theory of airfoil response in a gusty atmosphere. Part I. Aerodynamic transfer function. Tech. Rep. UTIAS Rep. No. 139. Institute for Aerospace Studies, University of Toronto.

Fleury, V., Bailly, C., Jondeau, E., Michard, M. \& Juvé, D. 2008 Space-time correlations in two subsonic jets using dual particle image velocimetry measurements. AIAA J. 46 (10), 2498-2509.

Fuchs, H. V. \& Michel, U. 1978 Experimental evidence of turbulent source coherence affecting jet noise. AIAA J. 16 (9), 871-872.

Graham, J. M. R. 1970 Similarity rules for thin aerofoils in non-stationary subsonic flows. J. Fluid Mech. 43 (4), 753-766.

HinZE, J. O. 1975 Turbulence. McGraw-Hill.

Landahl, M. 1961 Unsteady Transonic Flows. Pergamon.

LaU, J. C. \& Fisher, M. J. 1975 The vortex-street structure of turbulent jets. Part 1. J. Fluid Mech. 67 (2), 299-337.

Mish, P. F. \& Devenport, W. J. 2006 An experimental investigation of unsteady surface pressure on an airfoil in turbulence. Part 1. Effects of mean loading. J. Sound Vib. 296 (3), 417-446.

MoreaU, S. \& Roger, M. 2005 Effect of angle of attack and airfoil shape on turbulence-interaction noise. In Eleventh AIAA/CEAS Aeroacoustics Conference, Monterey, CA.

Moreau, S., Schram, C. \& Roger, M. 2007 Diffraction effects on the trailing edge noise measured in an open-jet anechoic wind tunnel. In Thirteenth AIAA/CEAS Aeroacoustics Conference, Rome, Italy.

Oerlemans, S. \& Migliore, P. 2004 Aeroacoustic wind tunnel tests of wind turbine airfoils. In Tenth AIAA/CEAS Aeroacoustics Conference, Manchester, UK.

OlsEN, W. A. 1976 Noise generated by impingement of turbulent flow on airfoils of varied chord, cylinders, and other flow obstructions. Tech. Rep. NASA-TM-X-73464.

Paterson, R. W. \& Amiet, R. K. 1976 Acoustic radiation and surface pressure characteristics of an airfoil due to incident turbulence. Tech. Rep. CR 2733. NASA.

Rockwell, D. \& NAUdASCHER, E. 1978 Review - self-sustaining oscillations of flow past cavities. J. Fluid Engng 100, 152-163.

Roger, M. \& Moreau, S. 2005 Back-scattering correction and further extensions of Amiet's trailing-edge noise model. Part I. Theory. J. Sound Vib. 286, 477-506.

Roger, M. \& Serafini, S. 2005 Interaction noise from a thin annulus in a circular jet. In Eleventh AIAA/CEAS Aeroacoustics Conference, Monterey, CA.

Ross, M. H., Shannon, D. W., Blake, W. K. \& Morris, S. C. 2008 Unsteady lift and radiated sound generated by a 2-d airfoil in a single stream shear layer. In Fourteenth AIAA/CEAS Aeroacoustics Conference, Vancouver, Canada.

Schram, C. 2003 Aeroacoustics of subsonic jets: prediction of the sound produced by vortex pairing based on piv. PhD thesis, Technical University Eindhoven ISBN 90-386-1605-8, Eindhoven, The Netherlands. 